\title{
Die Entwicklung des Fremdenverkehrs in der Stadt Kufstein bis 1914. Sommerfrische, Bergsport, Kur und die Anfänge des Wintertourismus
}

\author{
Tanja Angela Braunschmid \\ Kerngebiet: Österreichische Geschichte \\ eingereicht bei: ao. Univ.-Prof. Mag. Dr. Margaretha Friedrich \\ eingereicht im: SoSe 2019 \\ Rubrik: Bachelor-Arbeit
}

\begin{abstract}
The development of tourism in Kufstein until 1914. Summer and health tourism, alpine sports and the beginning of winter tourism

This bachelor thesis shows the economic importance of the touristic sector in Kufstein before the First World War. It examines how technical innovations, organisations and business-minded entrepreneurs developed the upcoming sector of summer tourism in Kufstein. The paper further discusses whether winter tourism already existed in Kufstein and whether this was already of economic importance for the small Tyrolean town.
\end{abstract}

\section{Einleitung}

Der Tourismus ist aus der Stadt Kufstein nicht mehr wegzudenken. Neben der Festung als Ausflugsziel und Hauptattraktion der Stadt sind vor allem die Kulturveranstaltungen Besuchermagnete. Die Stadt ist heute ganzjährig das Ziel von Tourist*innen, wobei sich anhand der Nächtigungszahlen aus dem Geschäftsbericht des Tourismusverbandes 2017 ein Vorteil in der Sommersaison zeigt: 52.843 Ankünften in der Sommersaison 2017 stehen 34.281 Ankünfte in der Wintersaison 2016/2017 gegenüber.' Die Anfänge des Tourismus in Tirol bzw. in Österreich im 19. Jahrhundert hängen mit der Sommer-

Tourismusverband Kufsteinerland, Geschäftsbericht 2017, 2017, [https://www.kufstein.com/media/ geschaeftsbericht-tourismusverband-kufsteinerland-2017.pdf], eingesehen 05.06.2018. 
frische und der Kur zusammen. Vor allem die Sommerfrische wird oft als Synonym für den Fremdenverkehr in der Monarchie verwendet. Aber was geschah in den Wintermonaten? Gab es im Winter in dieser Zeit keinen Fremdenverkehr? Die vorliegende Bachelorarbeit setzt an dieser Stelle an. Für die Untersuchung der Anfänge und der ersten Entwicklungen des Kufsteiner Fremdenverkehrs stellt sich die Frage, ob sich Kufstein, wie andere bekannte erste österreichische Fremdenverkehrsorte wie etwa Bad Ischl, ebenfalls als Sommerfrische- und Kur-Ziel etablieren konnte, oder ob der Fremdenverkehr in der Stadt möglicherweise mehr zu bieten hatte. Bei der Beschäftigung mit dem Fremdenverkehr in Kufstein ab der Mitte des 19. Jahrhunderts bis 1914 fallen immer wieder Dokumente und Fotografien aus den Wintermonaten auf. Konnte Kufstein also bereits während der Anfangsphase des Fremdenverkehrs auch in den Wintermonaten Gäste ansprechen?

Von diesem Interessenshintergrund ausgehend entstand folgende Forschungsfrage: Welche zentralen Entwicklungsschritte durchlief der Fremdenverkehr in Kufstein bis 1914, wodurch sich neben der Sommersaison auch bereits eine Wintersaison etablieren konnte? Die leitende These besagt also, dass Kufstein einer der wenigen Orte in Tirol war, denen es gelang, die Wintersaison vor 1914 zu einem bedeutenden wirtschaftlichen Faktor auszubauen. Besonders wichtige Entwicklungen, ohne die der Aufschwung des Fremdenverkehrs vor Beginn des Ersten Weltkriegs nicht möglich gewesen wäre, werden dabei dargestellt. Darunter fallen beispielsweise die Arbeit des Alpenvereins und die Erschließung des Kaisergebirges, der Ausbau der touristischen Infrastruktur, die Bedeutung der Eisenbahn sowie die aktiven Kufsteiner Förderer*innen, die an diesen neuen Wirtschaftsbereich glaubten. In der Betrachtung der Wintersaison vor 1914 geht es vor allem darum, die wirtschaftliche Relevanz herauszuarbeiten und zu zeigen, dass Kufstein bereits damals zu den Zwei-Saisonen-Tourismusorten zählte. Da diese Thematik in der bisherigen Forschung zur touristischen Entwicklung Kufsteins noch nicht berücksichtigt wurde, schließt die vorliegende Arbeit eine Forschungslücke.

Allgemeine Literatur zur Entwicklung des Fremdenverkehrs im heutigen Bundesland Tirol bildet die Grundlage für die Bearbeitung der Forschungsfrage. Zusätzlich werden Literatur und Quellen aus Kufstein selbst verwendet. Zeugnisse zur Entwicklung des Fremdenverkehrs in Kufstein finden sich beispielsweise in den Hotel- und Fremdenlisten des Vereins zur Hebung des Fremdenverkehrs in Kufstein und Umgebung, in Prospektmaterial von Gasthöfen und Hotels, und nicht zuletzt in zahlreichen Fotografien. Der Großteil der verwendeten Quellen wurde freundlicherweise vom Heimatverein Kufstein zur Verfügung gestellt. Ein herzliches Dankeschön dafür gilt besonders dem Obmann des Heimatvereins, Gerhard Lehmann.

Der bisherige Forschungsstand zum Thema behandelte vor allem Teilaspekte: 2016 erschien das Werk von Gerald Bendler mit dem Titel „Wilder Kaiser. Von Sommerfrischlern, Kletterlegenden, Skipionieren und dem Bergdoktor - 200 Jahre Alpingeschichte und Reisekultur"2. Diese Monografie beschreibt neben Inhalten zum alpinen Sport am Wil-

2 Gebhard Bendler, Wilder Kaiser. Von Sommerfrischlern, Kletterlegenden, Skipionieren und dem Bergdoktor. 200 Jahre Alpingeschichte und Reisekultur, Innsbruck-Wien 2016. 
den Kaiser weitere touristische Aspekte und bezeichnet Kufstein dabei als die Hauptstadt der Region. Der Kufsteiner Heimatverein lieferte 2012 und 2013 auf seiner Website einen Einblick in den Rodelsport. ${ }^{3}$ Eine umfassende Arbeit zur historischen Entwicklung des Fremdenverkehrs in der Stadt Kufstein vor 1914 scheint allerdings bisher zu fehlen.

Diesen Aspekten widmet sich vorliegende Arbeit: Nach der Betrachtung des Tourismus vor der zweiten Hälfte des 19. Jahrhunderts geht es um den Beginn des Sommertourismus und die Förderer*innen des Fremdenverkehrs in Kufstein. Dieser Teil schließt mit der Untersuchung des statistischen Materials hinsichtlich der Gästefrequenz und mit der Erkenntnis, dass sich Kufstein zu einem wichtigen Sommerreiseziel entwickeln konnte. Darauf folgt ein Kapitel zum beginnenden Wintertourismus. Der erste Abschnitt widmet sich der Frage, wann der Wintertourismus zeitlich begann. Ohne den Wintersport wäre eine Wintersaison vermutlich nicht möglich gewesen - daher werden zunächst der Wintersport und Skisport in Tirol allgemein betrachtet, ehe dann das Wintersportangebot in Kufstein und seine touristische Relevanz thematisiert werden. Am Ende dieses Kapitels wird anhand der Gästezahlen die Größenordnung des Wintertourismus deutlich, was Rückschlüsse auf die wirtschaftliche Relevanz dieser zweiten Saison zulässt.

\section{Die Frühgeschichte des Fremdenverkehrs und die Entzauberung der Berge}

In der Literatur besteht grundsätzlich Einigkeit darüber, dass der Tourismus in Tirol Mitte des 19. Jahrhunderts einsetzte. ${ }^{4}$ Engelbert Runggaldier identifizierte in seiner Dissertation für den touristischen Aufbruch zu dieser Zeit zwei wichtige Faktoren: Einerseits wurde durch Reiseschriftsteller*innen, allen voran durch den bekannten Schriftsteller Ludwig Steub, ein Interesse an und eine Sehnsucht nach Ferien in Nordtirol geweckt. Andererseits fällt in diesen Zeitraum auch der Bau der Eisenbahn. Damit war eine bessere Erreichbarkeit Tirols für breitere Schichten der Bevölkerung gewährleistet, wodurch die Eisenbahn als Wachstumsmotor für die touristische Entwicklung Tirols fungierte. ${ }^{5}$

Für Kufstein lässt sich kein genauer Startzeitpunkt für den Fremdenverkehr ermitteln. In einem Artikel aus dem Jahre 1931 wird der Beginn des Fremdenverkehrs für Anfang der 1870er-Jahre angesetzt. ${ }^{6}$ Dieser Schluss ist aus den folgenden Gründen glaubwürdig: Zwar setzte der Bau der Eisenbahn 1858 bereits einen wesentlichen Impuls für einen Zuwachs an Gästen in der Stadt Kufstein, erst ab 1870 ist aber eine vermehrte Institutionalisierung des Fremdenverkehrs erkennbar, u. a. durch die Gründung des Verschönerungsvereins oder durch die Gründung der Sektion Kufstein des Deutschen und

3 Heimatverein Kufstein, Rodeln vor 100 Jahren, o. D., [http://www.heimat-kufstein.at/2012/35-rodeln-vor-100jahren und http://www.heimat-kufstein.at/bildergalerie/category/38-0113-rodeln-v-100-jahren], eingesehen 15.10.2018.

$4 \quad$ Informationen zu dieser zeitlichen Einordnung des beginnenden Fremdenverkehrs finden sich u. a. bei Engelbert Runggaldier, Tirol und der Fremdenverkehr, Diss. Innsbruck 1927, S. 9-11.; Josef Riedmann, Geschichte Tirols, Wien 21988, S. 205-206; Roman Sandgruber, Ökonomie und Politik. Österreichische Wirtschaftsgeschichte vom Mittelalter bis zur Gegenwart (Österreichische Geschichte), Wien 1995, S. 284-285.

5 Runggaldier, Fremdenverkehr, S. 9-11.

6 Josef Blattl, Kufsteiner Fremdenverkehr in 60 Jahren, in: Eduard Lippott (Hrsg.), 60 Jahre Tiroler Grenzbote 18711931, Kufstein 1931, S. 18-19, hier S. 18. 
Österreichischen Alpenvereins. In diese Zeit fiel ebenfalls eine verstärkte touristische Bautätigkeit, wie etwa die Erbauung von Bad Kienbergklamm.

Auch vor Mitte des 19. Jahrhunderts zogen Reisende bereits durch Tirol. Nordtirol war dabei vorwiegend Durchzugsland, denn die Menschen im Mittelalter und der Frühen Neuzeit waren darum bestrebt, möglichst rasch zu ihren Zielen zu kommen. Neben Diplomat*innen waren vor allem Kaufleute auf den Hauptrouten, wie dem Inntal und dem Brenner, unterwegs. Primäre Reisemotive waren der Handel bzw. das Abwickeln von Geschäften.

Ein weiterer Reisegrund war die Wallfahrt, welche bereits eine frühe Form des Erholungsurlaubs und der Freizeitgestaltung darstellte. In den mehrtägigen Wallfahrten konnten die Bürger*innen aus ihrer gewohnten Umgebung und ihrem Alltag ausbrechen. Eine erste Blütezeit erreichte die Wallfahrt in Österreich um 1500, nach einer kurzen Unterbrechung zur Zeit der Reformation gewann sie in der Zeit der Gegenreformation und des Barock neuerlich an Auftrieb. Ab ca. 1830 stieg die Anzahl der Pilger*innen nochmals an. Dieser Aufschwung hing mit der katholischen Erneuerung und der zunehmenden Mobilisierung der Bevölkerung zusammen. ${ }^{8}$

Im 16. Jahrhundert bildete sich ein weiteres Reisemotiv aus: Reisen wurde vor allem in Adelskreisen als Teil der Bildung und Erziehung angesehen. Die sogenannte "Kavalierstour" oder "Grand Tour" begann und führte Söhne aus vornehmem Hause in die kulturell interessantesten Orte in Mitteleuropa und Italien. ${ }^{9}$ Ein ähnliches Phänomen war die bürgerliche Bildungsreise, die im gehobenen Bürgertum seit der Aufklärung auftrat. Auch bei dieser Form des Reisens ging es um eine Horizonterweiterung, wiederum waren wichtige Sehenswürdigkeiten, wie der Besuch antiker Kulturstätten, das Ziel. ${ }^{10}$ Tirol wurde in diesem Zusammenhang zwar nur auf der Durchreise gestreift, jedoch wurde die landschaftliche Schönheit bereits in Reiseberichten gewürdigt. ${ }^{11}$

All diese Formen des frühen Fremdenverkehrs berührten Tirol zwar nicht direkt, verhalfen aber dennoch zur Entstehung eines gewerblichen Gasthofwesens. Dieses etablierte sich im Laufe des 14. Jahrhunderts. Davor spielten Unterkünfte, die aus Gastfreundschaft unentgeltliche Beherbergung anboten, sowie Klosterherbergen und Unterbringungen in kirchlichen Hospizen und Pfarrhöfen eine zentrale Rolle. Die neu aufkommenden Gasthöfe boten nicht nur Fremden eine Unterkunft, sondern richteten sich mit ihrem Angebot auch an die Einheimischen. ${ }^{12}$ In Kufstein - als Grenzstadt wichtige Transit- und Mautstelle - gab es für die durchreisenden Gäste und die einheimische Bevölkerung

7 Paul Tschurtschenthaler, Der Tourismus im Bundesland Tirol 1918-1990, in: Anton Pelinka/Andreas Maislinger (Hrsg.), Handbuch zur neueren Geschichte Tirols (Zeitgeschichte 2), Innsbruck 1993, S. 113-208, hier S. 116.

8 Roman Sandgruber, Die Entstehung der Österreichischen Tourismusregionen, in: Andrea Leonardi/Hans Heiss (Hrsg.), Tourismus und Entwicklung im Alpenraum 18.-20. Jh. (Tourism \& Museum, Studienreihe des Touriseum 1), S. 201-226, hier S. 201-203.

9 Rüdiger Hachtmann, Tourismus-Geschichte, Göttingen 2007, S. 43-45.

10 Ebd., S. 48-49..

11 Tschurtschenthaler, Tourismus Tirol, S. 116.

12 Ebd., S. 117. 
schon früh verschiedene Wirtshäuser bzw. Gasthöfe. Einer der ersten war beispielsweise der Gasthof Arche Noe, der laut einer Postkarte seit 1650 bestand. ${ }^{13}$ Er befand sich direkt an der Anlegestelle der Innschifffahrt und war damit ideal für die Durchreisenden. Zu den ältesten Gasthöfen zählen die Gasthöfe in der Rennerhofgasse (heutige Römerhofgasse), wie das Auracher Löchl, welches bereits zu Beginn des 15. Jahrhunderts als Wirtshaus existierte. Auch am heutigen Unteren Stadtplatz standen sehr alte Gasthöfe, etwa der im 16. Jahrhundert gegründete Gasthof Auracher, der sich in einem Prospekt als eine der ältesten und renommiertesten Gaststätten in Tirol bezeichnet. ${ }^{14}$

Dass Kufstein aufgrund seiner Lage an einem Haupthandelsweg für Durchreisende ein gutes Angebot an Gaststätten vorweisen konnte, beschreibt auch Joseph Kyselak, ein reisender Beamter aus Wien: „In Kuffstein [sic!] sind [...] der Kommerzial-Straße wegen, genug Wirthshäuser vorhanden." ${ }^{\prime 15}$ Kyselak kam 1825 im Rahmen seiner ausgedehnten Fußreise durch Österreich und Bayern nach Kufstein und war somit ein Zeitzeuge für das frühe Beherbergungswesen vor dem ersten Aufschwung im Fremdenverkehr.

Es stellt sich an dieser Stelle nun die Frage, wie Tirol vom Durchzugsland zum eigentlichen Reiseziel werden konnte. Rüdiger Hachtmann bezeichnet die „Naturbegeisterung als Geburtshelfer des frühbürgerlichen Tourismus"16. Es war vor allem Jean-Jaques Rousseau, der dazu beitrug, dass sich eine Wandlung im Bewusstsein der Menschen vollzog: „Die bisherige Sicht der Alpen als unwirtliches, abstoßendes Gebirge wandelte sich allmählich hin zu ihrer Betrachtung als einer faszinierenden Kulisse von überwältigender Schönheit."17 Rousseaus Begeisterung für die Natur war nicht nur eine reine dichterische Schwärmerei, sie wurde zur Ideologie und förderte einen Einstellungswandel. So trat zu den oben genannten Reisemotiven ein neues und wesentliches Motiv für den Fremdenverkehr hinzu: der Wunsch nach Ruhe und Erholung in der Natur sowie die Sehnsucht nach dem Erleben der Naturschönheiten und Landschaften. ${ }^{18}$

Rousseau trug also maßgeblich zu einer Entwicklung bei, welche als Entzauberung der Berge bezeichnet werden kann:

„Die Alpen wurden von der bedrohlichen Naturbarriere, die den Weg nach Italien verstellt hatte und schwer zu überwinden gewesen war, zur schroffen Naturschönheit. Gleichwohl blieb das schaurig-schöne Panorama der Alpen angsteinflößend. Nun allerdings stieß die Gefahr nicht mehr ab; sie zog an. Gewitterstimmungen, überhängende Felsen und steile Abgründe - ähnlich wie an den Meeren der vorsichtige Blick über den Rand der Steilküste - machten jetzt die Faszination des Hochgebirges aus. Sie wurden zu Herausforderungen, die der Bergwanderer und Bergsteiger suchte, und brachen der,Touristik' im

13 Postkarte, 5.10.1937. Heimatverein der Stadt Kufstein, Fotosammlung Kufstein

14 Prospekt Gasthof zum „Auracher", o. D. (nach 1895) Innsbruck, S. 8.

15 Joseph Kyselak, Zu Fuss durch Österreich. Skizzen einer Wanderung nebst einer romantisch pittoresken Darstellung mehrerer Gebirgsgegenden und Eisglätscher unternommen im Jahre 1825 von Joseph Kyselak, nachgegangen und nachgedacht von Ernst Gehmacher, Wien u. a. 1982, S. 193.

16 Hachtmann, Tourismus-Geschichte, S. 59

17 Tschurtschenthaler, Tourismus Tirol, S. 117

18 Hachtmann, Tourismus-Geschichte, S. 60. 
ursprünglichen Sinne des Wortes - anfangs gleichbedeutend mit,Alpinistik' Bahn."19

Der Wunsch nach dem freizeitlichen Natur- bzw. Bergerlebnis wurde vom gesellschaftlichen und sozialen Wandel verstärkt: Die Menschen sehnten sich in einer Zeit der zunehmenden Verstädterung und des erhöhten Arbeitseinsatzes nach Erholung in der intakten Natur. Gleichzeitig führte ein zunehmender finanzieller Besserstand dazu, dass sich mehr Menschen eine solche Erholungsreise leisten konnten. ${ }^{20}$

Die steigende touristische Nachfrage in Tirol schien eng verbunden mit einigen Schriftsteller*innen, die zum neuen Ruhm des Landes als Reiseziel beitrugen und somit erste Botschafter*innen für den Fremdenverkehr waren. An dieser Stelle sei der Tiroler Schriftsteller Beda Weber genannt, der in den 1840er-Jahren in drei Bänden über das Land Tirol schrieb und darauf aufbauend 1853 ein Handbuch für Reisende in Tirol verfasste. ${ }^{21}$ Von besonderer Bedeutung war Ludwig Steub aus München. Neben verschiedenen Novellen über Tirol hinterließ er das Werk "Drei Sommer in Tirol", welches 1846 erschien und untrennbar mit der weiteren touristischen Entwicklung Tirols verbunden ist. Noch 50 Jahre später wurde in einem Nachruf in der touristischen Zeitschrift "Der Alpenfreund" darauf hingewiesen, dass Steub wesentlich dazu beigetragen hatte, dass Personen auf Tirol als Reiseziel aufmerksam wurden und das Land Tirol ihm dafür dankbar sein sollte.22

Steub wie auch Weber schilderten ihrer Leserschaft die Naturschönheiten und klimatischen Vorzüge Tirols und weckten zudem die Neugier auf die Kultur und die Eigenarten der Bevölkerung. In der Literatur taucht der Name Steub in Zusammenhang mit der Fremdenverkehrsentwicklung häufig auf. Auch Runggaldier findet in seiner Dissertation lobende Worte für die Arbeit Steubs:

„Der Name Steub ist auch mit der weiteren Entwicklung des Fremdenwesens in unserem Lande eng verflochten, denn es verging kein Jahr, ohne dass nicht ein Buch oder mindestens größere Aufsätze in Zeitungen von diesem Schriftsteller über Tirol erschienen wären, worin insbesondere unsere sozialen Zustände lebensvoll, wahrheitsgetreu und in anziehender, zumeist mit köstlicher Laune gewürzter Darstellung geschildert wurden."23

Der aus München stammende Heinrich Noë war ebenfalls als Reiseschriftsteller ein wichtiger Schilderer der Alpen. ${ }^{24}$ Sowohl Steub als auch Noë sollen auf ihren Reisen nach Kufstein gekommen sein. ${ }^{25}$

19 Hachtmann, Tourismus-Geschichte, S. 62.

20 Klaus Planer, Der Fremdenverkehr Nordtirols, Diss. Wien 1970, S. 260.

21 Beda Weber, in: Wikipedia. Die freie Enzyklopädie, aktualisiert am 21.12.2017, [https://de.wikipedia.org/wiki/ Beda_Weber], eingesehen 27.5.2018.

22 Alfred Lunglmayr, Drei Sommer in Tirol, in: Der Alpenfreund. Illustrierte Touristenzeitschrift für das Alpengebiet 2 (1896), Heft 12, S. 1-4, hier S. 4.

23 Runggaldier, Fremdenverkehr, S. 11.

24 Heinrich Noë, in: Wikipedia. Die freie Enzyklopädie, aktualisiert am 2.10.2018, [https://de.wikipedia.org/wiki/ Heinrich_Noë], eingesehen 10.10.2018.

25 Zu lesen in den Erinnerungen an Dekan Dr. Matthäus Hörfarter von Paul Weitlaner. Steub und Noë seien Gäste des Auracher gewesen: Paul Weitlaner, Dekan Dr. Matthäus Hörfarter, in: Franz Biasi (Hrsg.), Kufsteiner Buch. Beiträge zur Heimatkunde von Kufstein und Umgebung, Bd. 1 (Schlern Schriften 156), Innsbruck 1957, S. 121-138, hier S. 135. 


\section{Der Beginn des Sommertourismus und die Förderer*innen des touristi- schen Aufbruchs}

Im Reiseführer „Kufstein und seine Umgebung. Ein Führer für Fremde und Einheimische” aus dem Jahr 1904 fanden die im vorhergehenden Kapitel beschriebenen Entwicklungen - der Einfluss von Rousseau und Steub auf den Aufschwung für Reisen nach Tirol ebenfalls eine Erwähnung. ${ }^{26}$ Auch die bestehenden Wirtshäuser und die verkehrsgünstige Lage Kufsteins als Grenzstadt wurden aufgegriffen:

„Dort, wo sich der Inn der Fesseln entledigt, mit welchen die Berge seine grünen Fluten umschlingen, war schon immer eine Hauptpforte für den tirolischen Verkehr. Auch der Fremdenstrom kam von dieser Seite und stieß auf das Grenzstädtchen Kufstein, welches imstande ist, dem Fremden ein kleines Bild des ganzen Landes zu geben, indem es auf einem kleinen Raume alles in sich verewigt, was Tirol dem Besucher zu bieten vermag. Kufstein konnte daher von den Touristen und Sommerfrischlern nicht übersehen werden und war einer der ersten Plätze, welche einen ständigen Fremdenverkehr nachweisen konnte. Es fehlte in dem fortschrittlich gesinnten Städtchen nicht an Männern, welche weitausblickend die Vorteile des Fremdenverkehrs, dieses, Exportes in den Magen der Fremden', erkannten und nichts ungetan ließen, diese Einnahmsquelle dem Volke dauernd zu erhalten. Hier ist vor allem der Gründer der Sektion Kufstein des D. u. Ö.-A.V. hochw. Herr Dekan Dr. Math. Hoerfarter zu erwähnen, der selbst den Luftkurort und das Bad Kienbergklamm erbaute, Wege und Pflanzungen anlegte $[\ldots]^{\prime \prime 27}$

Die Förderer*innen des Kufsteiner Fremdenverkehrs zusammen mit der technologischen Entwicklung der Eisenbahn sind Thema dieses Kapitels.

\subsection{Die Eisenbahn als Motor für den Fremdenverkehr}

Die technologische Entwicklung der Eisenbahn machte es möglich, dass die Erholungssuchenden nicht mehr in der Nähe ihrer Heimatorte ihren Aufenthalt verbringen mussten, sondern mühelos weitere Distanzen zurücklegen konnten. ${ }^{28}$ Das Reisen wurde durch die Bahn komfortabler, kürzer und weniger beschwerlich. Dadurch wurde der Fremdenverkehr allmählich zur ökonomisch relevanten Größe, wodurch die Eisenbahn als ein Fremdenverkehrsmotor bezeichnet werden kann. Der Planung und dem Bau der Eisenbahn durch Tirol war ein langes Bemühen des Tiroler Landtags vorangegangen: Bereits 1836 wandte sich dieser zum Thema der Tiroler Bahnstrecke an Wien und legte 1838 den ersten Entwurf für die Strecke zwischen Kufstein und Innsbruck vor. ${ }^{29} 1847$ erfolgte die Aufnahme des geplanten Streckenbaus durch Tirol in die offizielle Planung

\footnotetext{
26 Verein zur Hebung des Fremdenverkehrs in Kufstein und Umgebung (Hrsg.), Kufstein und seine Umgebung. Ein Führer für Fremde und Einheimische, Kufstein 1904, S. 57.

29 Benjamin Türkis, Innsbrucker Tourismusgeschichte (Geschichte und Ökonomie 20), Innsbruck 2010, S. 12.
} 
für den Eisenbahnbau. Es wurde ein Zusammenschluss der deutschen und österreichischen Strecken bei Passau, Salzburg und Kufstein beschlossen. ${ }^{30}$

Im August 1853 genehmigte Kaiser Franz Joseph den Bahnbau, woraufhin die Bauarbeiten im September desselben Jahres begannen. Die Arbeiten erfolgten zeitgleich von zwei Seiten: In Tirol wurde mit dem Bau des Teilstücks von Innsbruck in Richtung Kufstein begonnen, gleichzeitig baute Bayern die Strecke von Rosenheim (bzw. München) nach Kufstein. ${ }^{31} 1858$ schließlich wurde die erste Eisenbahnstrecke in Tirol, welche bereits durch Kufstein führte, eröffnet. Zunächst erfolgte eine Anbindung an das Deutsche Reich. So fuhr am 4. August 1858 der erste Zug zwischen Rosenheim und Kufstein. Es folgte dann die Strecke Innsbruck-Kufstein mit offiziellem Start am 24. November 1858. Damit trat Tirol in das Bahnzeitalter ein.32

Neben der neuen Erreichbarkeit von Ortschaften an der Bahnstrecke und der Verkürzung der Reisezeiten brachte die Bahn auch vielerorts ein moderneres Beherbergungswesen, denn es entstanden vielfach Grand Hotels in Bahnhofsnähe. Diese Hotels wurden von den Bahngesellschaften selbst errichtet. ${ }^{33}$ In Kufstein war dies zwar nicht der Fall, aber ein Hotelneubau stand doch mit dem Eisenbahnbau in direkter Verbindung. Es handelte sich um das Hotel Gisela, welches 1877 eröffnet wurde und noch heute direkt gegenüber dem Bahnhofsgebäude steht. Die Namenswahl für dieses neue Hotel stand in Zusammenhang mit der Eisenbahn: Als "Giselabahn“ war die Eisenbahnstrecke von Salzburg über Bischofshofen und Kitzbühel nach Wörgl bekannt. Dem Kaiser und dem wirtschaftlich so bedeutenden Bahnbau zu Ehren wurde das Hotel nach Gisela von Österreich benannt, der zweiten Tochter von Kaiser Franz Joseph I. und Kaiserin Elisabeth. ${ }^{34}$

Für die weitere Entwicklung des Fremdenverkehrs in Kufstein dürfte die Verschiebung der Hauptverkehrsrouten besonders relevant gewesen sein. Zwar lag Kufstein an einem Handelsweg und hatte auch bisher schon als Grenzstadt eine wichtige Bedeutung, aber dennoch führte der schnellste Weg, zumindest im Personenverkehr, aus dem Norden über das Königreich Bayern in Richtung Innsbruck und dann weiter nach Italien nicht durch das Inntal, sondern über Garmisch-Partenkirchen oder Mittenwald und Zirlerberg. ${ }^{35}$ Mit der Eisenbahn änderte sich das: Der schnellste und bequemste Weg führte nun über Kufstein nach Innsbruck. Es ist anzunehmen, dass dieser neue Durchreiseverkehr mit Grenzkontrolle und Zollabfertigung der Stadt Kufstein als Fremdenverkehrsort möglicherweise zu einer höheren Bekanntheit verholfen hat.

Die Eisenbahnstrecken lenkten gewissermaßen die Nachfrage der Reisenden. Mit der Lage an einer der Hauptverkehrsrouten und aufgrund der Verbindung nach Bayern war Kufstein geradezu prädestiniert, ein Sommerfrischeort für die Städter*innen aus Mün-

30 Benjamin Türkis, Innsbrucker Tourismusgeschichte (Geschichte und Ökonomie 20), Innsbruck 2010, S. 12.

31 Eduard Lippott, Kufsteiner Chronik 1788-1918, in: Franz Biasi (Hrsg.), Kufsteiner Buch. Beiträge zur Heimatkunde von Kufstein und Umgebung, Bd. 2 (Schlern Schriften 157), Innsbruck 1958, S. 9-142, hier S. 49.

32 Ebd., S. 50

33 Sandgruber, Ökonomie und Politik, S. 286.

34 Den Hinweis zur Namenswahl kam vom Heimatverein Kufstein. Auf das Eröffnungsjahr des Hotels verweist die hoteleigene Homepage. Hotel Gisela Kufstein, o. D., [https://www.hotelgisela.at/], eingesehen 16.08.2018.

35 Bendler, Wilder Kaiser, S. 18-19. 
chen zu werden. ${ }^{36}$ Aus den Fremden- und Wohnungslisten geht hervor, dass der Großteil der Gäste vor Beginn des Ersten Weltkriegs aus dem Königreich Bayern bzw. aus den Großstädten des heutigen Deutschlands, wie etwa aus Berlin, nach Kufstein kam.

Wie zentral die Eisenbahn für die Entwicklung des Fremdenverkehrs in Kufstein war, verdeutlichen Inserate oder Werbeprospekte der Kufsteiner Hotels. Die Distanz zum Bahnhof durfte dabei als Information nicht fehlen. Außerdem war der Fahrplan der Eisenbahn in jeder "Fremden- \& Wohnungsliste für Kufstein und das Kaisertal" enthalten. Auch im Reiseführer "Kufstein und seine Umgebung" von 1904 wurde gleich einleitend auf die gute Erreichbarkeit per Bahn und vor allem auf die Bedeutung von München für den Kufsteiner Fremden- bzw. Ausflugsverkehr hingewiesen:

„Die liebliche, in rastlosem Aufstreben befindliche, tirolische Grenzstadt Kufstein kann von mehreren Seiten erreicht werden, am bequemsten natürlich an jenen ,Einbruchsstellen', die durch die Eisenbahnen und Heeresstrassen hergestellt werden. Hier kommt zunächst in Betracht der Zugang vom Deutschen Reiche: 1. München - Rosenheim - Kufstein. Auf dieser Strecke verkehren namentlich im Sommer so viele Züge, dass man zu jeder Zeit bequem hin- und zurückgelangen kann. Besonders günstig ist es für die Münchner Ausflügler, die mit dem Schnellzuge in guten 1 1 1/2, mit dem Postzuge in 2 1 1/4 Stunden und vom Ostbahnhof in je um $1 / 4$ Stunde weniger Zeit nach Kufstein kommen und von da aus das herrliche Kaisertal besuchen können." ${ }^{\text {37 }}$

\subsection{Dekan Dr. Matthäus Hörfarter - ein touristischer Pionier für Kufstein}

Dekan Dr. Matthäus Hörfarter, der 1859 seine seelsorgerische Tätigkeit in Kufstein begann, war für die Stadt weit mehr als nur ein Seelsorger. ${ }^{38}$ Er war ein Förderer und Visionär für den Kufsteiner Fremdenverkehr und maßgeblich an dessen Ausbau und Institutionalisierung beteiligt.

Bereits während seines Studiums in Salzburg entdeckte er seine Liebe zum Alpinismus und machte erste Erfahrungen in der Bergwelt, die wegweisend für seine späteren Bemühungen in Kufstein waren. ${ }^{39}$ In seiner Freizeit ging er seiner Leidenschaft für das Wandern nach und war viel im Kaisergebirge unterwegs. Im Nachruf von Rudolf Sinwel aus dem Jahre 1899 wird er als leidenschaftlicher Berg- und Naturfreund dargestellt, der diese Begeisterung für die Bergwelt seinen Mitmenschen näherbringen wollte: „Es war ihm ein Herzensbedürfnis, auch seinen Mitmenschen die Freude an der Natur einzuflößen und sie zum Naturgenusse anzuregen." ${ }^{40}$ Dieses Bedürfnis führte zusammen mit der Er-

36 Sandgruber, Ökonomie und Politik, S. 286.

37 Verein zur Hebung des Fremdenverkehrs, Kufstein, S. 10.

38 Hörfarter ist vor allem durch seine Bemühungen auf dem Gebiet der Kindergartenpädagogik und Schulbildung bekannt. 1870 wurde auf sein Bemühen hin in Kufstein der erste Kindergarten Tirols eröffnet. Nur zwei Jahre darauf begründete Hörfarter die Kindergärtnerinnenausbildung. Mehr Informationen finden sich bei: Weitlaner, Hörfarter, S. 132.

39 Ebd., S. 121-122.

40 Rudolf Sinwel (Hrsg.), Dr. theol. Matth. Hoerfarter. Gedenkschrift zur Enthüllung des Hoerfarter-Denkmales in Kufstein am 10. September 1899, Kufstein 1899, S. 36. 
kenntnis, dass für die weitere Stadtentwicklung der Fremdenverkehr eine wirtschaftlich wichtige Rolle spielen werde, zu verschiedensten Bemühungen Hörfarters rund um den Fremdenverkehr. Hörfarter kann daher durchaus als Kufsteiner Fremdenverkehrspionier bezeichnet werden. Die Gründung des Verschönerungsvereins und die Erbauung von Bad Kienbergklamm sind Teil dieser Pionierarbeit und werden in den folgenden Unterkapiteln ausgeführt.

Wie wichtig sein Wirken für die Stadt Kufstein gewesen ist, unterstreicht beispielsweise die Tatsache, dass im eingangs zu diesem Kapitel angeführten Zitat aus dem Reiseführer Hörfarters Wirken für den Fremdenverkehr in Kufstein auch 1904 noch als wesentlich hervorgehoben wurde. ${ }^{41}$ In einem Rückblick auf die ersten Jahre des Fremdenverkehrs im Artikel „Kufsteiner Fremdenverkehr in 60 Jahren“ in einer Sonderausgabe des „Tiroler Grenzboten" aus dem Jahre 1931 wurde Hörfarter ebenso als Pionier des Fremdenverkehrs gewürdigt. ${ }^{42}$

\subsection{Verschönerungsverein, Alpenvereinssektion und der erste Landesverband in Tirol}

Hörfarters erste Handlung auf dem Gebiet des Fremdenverkehrs war die Gründung des Verschönerungsvereins 1874. Ziel des Vereins war vor allem die Hebung der landschaftlichen Schönheit der Stadt und der näheren Umgebung durch das Anlegen von Wegen, Alleen und Baumbepflanzungen. ${ }^{43}$ Kufstein war damit einer der ersten Orte, die einen solchen Verschönerungsverein vorweisen konnten. Nur Meran, Arco und Gries bei Bozen mit den Kurkommissionen sowie Bruneck und Hall hatten schon früher eine entsprechende Institution. ${ }^{44}$ Die Verschönerungsvereine und Kurkommissionen waren die ersten Organisationsformen im Tiroler Fremdenverkehr. Adolf Lässer verweist darauf, dass der Zeitpunkt der Gründung einer solchen Organisation für den Fremdenverkehr von verschiedenen Faktoren abhängig war: Die Anzahl der Gäste und der Beherbergungsbetriebe und nicht zuletzt die Initiative durch Persönlichkeiten in den Fremdenverkehrsorten waren ausschlaggebend für eine frühe Gründung. ${ }^{45}$

Die „Kufsteiner Chronik“ belegt anhand verschiedener Einträge, welche zum Beispiel auf neu angelegte Wege oder Alleen hinweisen, die Aktivität des Verschönerungsvereins. Im Eintrag zum Jahr 1882 findet sich eine recht detaillierte Information aus dem Jahresbericht des Vereins:

„Der Jahresbericht war erfreulich. Es wurden 13 Ruhebänke an öffentlichen Plätzen aufgestellt, Wegzeiger am Stadtberg, Brentenjoch, Eiberg und Hinterstein errichtet, in Kindsbündl Ruhesitze und Anlagen errichtet. Ein schöner Weg wurde über den Kienberg bis zur Duxeralpe angelegt, der Platz vor dem Schulhause

\footnotetext{
41 Verein zur Hebung des Fremdenverkehrs, Kufstein, S. 57-58.

42 Blattl, Fremdenverkehr, S. 18.

43 Sinwel, Hoerfarter, S. 36.

44 Adolf Lässer, 100 Jahre Fremdenverkehr in Tirol. Die Geschichte einer Organisation (Tiroler Wirtschaftsstudien 40), Innsbruck 1989, S. 19.

45 Lässer, Fremdenverkehr, S. 14.
} 
freigelegt, mit Kastanienbäumen und Bänken versehen, ebenso bei der alten Schießstätte ein angenehmer Aufenthalt für alt und jung geschaffen." ${ }^{46}$

Die Erschließung der Gebirgswelt war ebenfalls ein Anliegen Hörfarters. Am 24. Januar 1877 gründete er daher die Sektion Kufstein des Deutschen und Österreichischen Alpenvereins und stand dieser selbst 13 Jahre vor. Für die touristische Erschließung des Kaisergebirges waren der Verein und die damit verbundene Erbauung von Hinterbärenbad, der ersten Unterkunftshütte im Kaisertal, von wesentlicher Bedeutung. ${ }^{47}$

Trotz der steigenden Gästezahl in Kufstein, die wohl auf die neuen Institutionen zurückzuführen war, dürften Hörfarters Bemühungen in der Stadt nicht ganz unumstritten gewesen sein. Wie bei Rudolf Sinwel zu lesen ist, schienen vor allem Bitten um monetäre Förderung auf wenig Verständnis zu stoßen:

"Diese beiden Körperschaften [gemeint sind der Verschönerungsverein und die Alpenvereinssektion, Anm. d. Verf.] hatten anfangs keine leichte Arbeit in der Bevölkerung, der für Naturschönheit und Alpinistik noch das Verständnis fehlte. Gab es doch Kufsteiner genug, die sie als lästige Bettelvereine bezeichneten. Ja, einzelne Käuze mag es wohl heute noch geben, die auf diesem spießbürgerlichen Standpunkte stehen. Aber laut wagen sie es nicht mehr zu sagen angesichts der großartigen Erfolge und der allgemeinen Anerkennung ihres verdienstlichen Wirkens." ${ }^{48}$

Hörfarters Wirken blieb nicht auf Kufstein beschränkt. Seine Bemühungen rund um den Fremdenverkehr waren über die Stadt hinaus in Tirol bekannt, weshalb er in die Gründung einer ersten Organisation für den Fremdenverkehr in Nordtirol involviert wurde. Im Februar 1889 gab es eine Versammlung von Persönlichkeiten des Nordtiroler Fremdenverkehrs, in welcher die mögliche Gründung eines Verbands zur Förderung des Fremdenverkehrs thematisiert wurde. Hintergrund dafür war, dass es bereits im Januar zur Gründung eines Deutsch-Süd- und Osttiroler Fremdenverkehrsvereins in Brixen gekommen war und nun auch Nordtirol diesem Beispiel folgen wollte. Auf dieser ersten Versammlung wurde beschlossen, dass es zur Gründung eines Verbandes kommen sollte. Ein Komitee, bestehend aus Gastwirt*innen und Förderer*innen des Fremdenverkehrs in Nordtirol - darunter auch Hörfarter, bereitete einen Entwurf für die Statuten vor, um in der Folge eine konstituierende Hauptversammlung abzuhalten. Nach dem Abschluss der Vorbereitungsarbeiten kam es am 16. April 1889 zur eigentlichen Gründungsversammlung für den Verein zur Hebung des Fremdenverkehrs für Nordtirol. Hörfarter wurde in den ersten Ausschuss gewählt und war somit an der ersten landesweiten Fremdenverkehrsorganisation beteiligt. ${ }^{49}$ 


\subsection{Bad Kienbergklamm}

Neben seinen Tätigkeiten zur Institutionalisierung war auch die Erbauung von Bad Kienbergklamm von großer Wichtigkeit für die Entwicklung des Fremdenverkehrs in Kufstein. Bereits seit dem späten 18. Jahrhundert war das Thema Bad und Kur aus touristischer Sicht relevant geworden. Ärzte begannen aus gesundheitlichen Gründen, Bäder zu empfehlen. Nachdem erste Erfolge durch Heilbäder - beispielsweise in Bad Ischl durch die Ischler Sole - an Kranken bekannt wurden, entwickelte sich eine neue Badekultur. Zunächst waren es Adelige, die sich auf Kuraufenthalten erholten. Doch ähnlich wie bei der Sommerfrische ging der Kuraufenthalt im Laufe des 19. Jahrhunderts in die soziale Breite. Da neben dem gesundheitlichen Angebot an Bädern das gesellschaftliche Vergnügen immer wichtiger wurde, etablierten sich die Kuranwendungen während der Sommerfrische. ${ }^{50}$

Wie im folgenden Zitat von Hörfarter ersichtlich wird, sollte das Bad den Erholungsbedarf der städtischen Sommergäste in Kufstein decken:

„Im Jahre 1876 stand am Eingang der Kienbachklamm nur ein kleines Häuschen, von einer achtzigjährigen Jungfrau als Eigenthümerin bewohnt. Ein schlechter Weg, nur von Ziegen betreten, führte daran vorüber in den Berg hinan; Menschen kümmerten sich wenig um diese öde Stätte. [...] Diese wilde Steingrube sollte in einen angenehmen Sommer-Aufenthalt für die durch Studien und Berufsarbeit ermüdeten Städter umgewandelt werden, wo sie im Genuss der kostbaren Bergluft und im harzigen Duft des Nadelholzes die erlahmten Kräfte wieder erfrischen und im gemüthlichen sorgenlosen Beisammenleben den gesunkenen Lebensmuth wieder finden könnten. [...] Manche schüttelten bedenklich den Kopf, als man zuerst Hand anlegte, den wilden Steingraben zu cultivieren. Aus der Tiefe heraus, hart am tosenden Wildbach musste der Grund für die Bäder gelegt werden. [...] Um ein anständiges Haus zur Aufnahme für Fremde herzustellen, musste der Berg abgegraben, der Boden geebnet werden. [...] Das Bedürfnis, einen Sammelpunkt für die vielen Fremden zu schaffen, gab die Veranlassung zum Bau des Cursalons. Aber in der engen Thalschlucht fand sich kein Platz mehr; er musste dem Berg durch Felsensprengung abgetrotzt werden. [...] Im dritten Jahre erstand an erhöhter Stelle ein Glashaus mit Belvedere im Schweizerstil darüber."

In drei Jahren Bauzeit von 1876 bis 1879 wurde eine Kurinfrastruktur geschaffen, welche der Erwartungshaltung der Gäste entsprach. Neben dem Bad wurden eine Allee sowie Spazierwege angelegt und die eigentliche Kienbergklamm wurde durch Holzstege zum Überqueren erschlossen. ${ }^{52}$

Vom neuen Bad profitierte nicht nur Bad Kienbergklamm selbst, sondern es entwickelte sich eine Art kleiner Luftkurort. So wird in Inseraten aus den Fremdenlisten und

50 Sandgruber, Tourismusregionen, S. 206.

51 Sinwel, Hoerfarter, S. 37-38.

52 Ebd., S. 38-39. 
dem Reiseführer von 1904 Bad Kienbergklamm als Luftkurort bezeichnet. ${ }^{53}$ Das im Zitat Hörfarters erwähnte Häuschen wurde in das Hotel Garni umgewandelt und die Gäste mieteten sich sowohl im Hotel Bad Kienbergklamm als auch in den benachbarten Häusern und Villen ein. Im Nachruf auf das Werk Hörfarters ging Rudolf Sinwel sogar so weit, dass er den Bau von Bad Kienbergklamm als Anstoß für ein neues unternehmerisches Denken für den Fremdenverkehr bezeichnete, der weitere Hotelbauten und Investitionen in die touristische Infrastruktur ausgelöst haben soll. ${ }^{54}$ Zwar ist dieses Lob vermutlich übertrieben und auf das Bestreben zurückzuführen, Hörfarter im Nachruf in das bestmögliche Licht zu rücken, aber dennoch war Bad Kienbergklamm tatsächlich von großer Bedeutung für die Fremdenverkehrsentwicklung in Kufstein: Es begründete den Ruf Kufsteins als Luftkurort, und das Angebot für den Sommergast wurde durch diese zusätzliche Infrastruktur aufgewertet. Bad Kienbergklamm wurde im Fremdenführer von 1904 beispielsweise besonders gewürdigt, da es diesem Fremdenführer zufolge „die Aera des regen Fremdenverkehres für Kufstein eröffnete“" ${ }^{\prime \prime}$. Da für diese frühen Jahre des Fremdenverkehrs noch keine Statistiken vorliegen, lässt sich nicht feststellen, ob diese Behauptung der Wahrheit entspricht.

\subsection{Der Alpenverein und die Bedeutung des Kaisergebirges}

Neben Sommerfrische und Kur waren der beginnende Alpinismus und die Sehnsucht der Gäste nach dem Erlebnis der Bergwelt die Hauptwachstumsmotoren für den Tiroler Fremdenverkehr vor dem Ersten Weltkrieg. Nachdem die Bergwelt, wie bereits im zweiten Kapitel angesprochen, ihren Schrecken verloren hatte, wurden die Alpen zunehmend bestiegen. Vor allem Vertreter*innen der städtisch-intellektuellen Welt wagten sich an Erstbesteigungen, begeisterten sich für den Alpinismus und bemühten sich darum, das Gebirge für ein breiteres Publikum zu erschließen.

1857 gründeten englische Bergsteigerpioniere mit dem British Alpine Club den ersten alpinen Verein. Die aristokratischen Mitglieder dieses Sportvereins begannen erstmals mit einer systematischen Erschließung der Alpen und waren somit wegweisend für die Folgevereine. 1862 gründeten Akademiker, welche vorwiegend einen wissenschaftlichen Zugang zu den Bergen hatten, in Wien den Österreichischen Alpenverein. ${ }^{56}$ Neben einer leichteren Bereisung des Gebirges lag das Ziel der Vereinigung laut den ersten Statuten vor allem darin, die Kenntnisse über die Alpen zu erweitern und die Liebe zu den Bergen zu fördern. Kartografie und die Dokumentation der Tiere und Pflanzen waren dabei die Hauptanliegen. Demgegenüber standen einige Männer, unter ihnen der Tiroler Franz Senn, die für eine praktische Erschließung der Alpen eintraten. Daher wurde 1869 in München der Deutsche Alpenverein gegründet, welcher sich vorwiegend diesem Zweck verschrieb. ${ }^{57}$ Neben Franz Senn waren Johann Stüdl aus Prag sowie die beiden Münchner Theodor Trautwein und Karl Hofmann die Gründungsväter dieses

53 Verein zur Hebung des Fremdenverkehrs, Kufstein, Inseratenteil S. 7.

54 Sinwel, Hoerfarter, S. 39.

55 Verein zur Hebung des Fremdenverkehrs, Kufstein, S. 72.

56 Bendler, Wilder Kaiser, S. 40

57 Türkis, Tourismusgeschichte, S. 11. 
Vereins. Nur wenige Jahre später, im Jahre 1873, schlossen sich die beiden bestehenden Vereine zum Deutsch-Österreichischen Alpenverein zusammen. ${ }^{58}$ Damit war der Grundstein für die systematische und praktische Erschließung der Alpen gelegt. Durch den Bau von Wegen und Schutzhütten und durch zahlreiche Publikationen wurde ein breiteres Publikum auf die Alpen als Orte des Sports und der Erholung aufmerksam. ${ }^{59}$ Die Alpenvereine verbreiteten somit den Alpinismus, trugen wesentlich zur Vermarktung der Alpen bei und erleichterten das Wandern.

Vor allem der Alpenvereinsgründer Karl Hofmann spielte für das Kaisergebirge und damit für die spätere Gründung der Alpenvereinssektion Kufstein eine wichtige Rolle. Hofmann, der sich sehr für das Kaisergebirge interessierte, kam im Sommer 1869 mehrfach in das Gebiet, um die höchsten Gipfel zu erwandern und deren Höhe zu bestimmen. Dabei behielt er seine Begeisterung nicht für sich, sondern veröffentlichte seine Erfahrungen im ersten Jahrbuch des Deutschen Alpenvereins. Allen voran wurde dadurch die Bevölkerung aus dem Münchner Raum auf das Kaisergebirge aufmerksam. Nach dem Zusammenschluss zum Deutschen und Österreichischen Alpenverein (DuOeAV) war besonders die Sektion München am Kaisergebirge und dessen Erschließung für den Fremdenverkehr interessiert. Diese Sektion war es auch, welche die Gründung einer eigenen Kufsteiner Sektion anregte und sich mit dieser Idee an Dekan Hörfarter in seiner Funktion als Vorstand des Verschönerungsvereins wandte. Hörfarter gefiel dieser Gedanke und er sah in der touristischen Erschließung des Kaisergebirges Vorteile für den Fremdenverkehr in Kufstein. Er bemühte sich in der Stadt um weitere Anhänger*innen für diese Idee und gewann 37 Mitglieder bestehend aus prominenten Kufsteiner Persönlichkeiten, darunter Gastwirte und Hotelbesitzer, aber auch Kaufmänner und Lehrer. ${ }^{60}$

Am 24. Jänner 1877 wurde die Sektion Kufstein des DuOeAV gegründet. Die neue Sektion setzte sich vor allem die leichtere Bereisung des Kaisergebirges zum Ziel. Dies sollte einerseits durch die Organisation des Bergführerwesens und durch den Bau von Wegen und Unterkünften erreicht werden. Andererseits sollten Publikationen und Informationen herausgegeben werden. Die Ziele und Absichten des Alpenvereins deckten sich somit weitgehend mit den Interessen der Fremdenverkehrsentwicklung. In einem Schreiben an den Zentral-Ausschuss des DuOeAV nach der Gründung der Sektion Kufstein wies Hörfarter darauf hin, dass es eine enge Zusammenarbeit zwischen Verschönerungsverein und Alpenvereins-Sektion geben sollte. ${ }^{61}$ Dies war insofern möglich, da Hörfater selbst als Vorstand des Verschönerungsvereins und der Alpenvereins-Sektion fungierte. Die Tätigkeit beider Vereine war klar auf die Fremdenverkehrsentwicklung ausgerichtet. Dass Hörfarter als Fremdenverkehrs-Pionier auch an der Gründung des Alpenvereins beteiligt war, erscheint nicht ungewöhnlich. Adolf Lässer kommt in seinen Recherchen zu folgendem Schluss: „Zahlreiche Persönlichkeiten, die sich des Fremdenverkehrs in den Tiroler Orten und Tälern annahmen, sind auch Gründungsmitglieder der AV-Sektion [...].“62

\footnotetext{
58 Lässer, Fremdenverkehr, S. 29.

59 Türkis, Tourismusgeschichte, S. 11-12.

60 Bendler, Wilder Kaiser, S. 41-43.

61 Ebd., S. 44

62 Lässer, Fremdenverkehr, S. 30.
} 
Für die Erschließung des Kaisergebirges als Gebirgsattraktion von Kufstein waren vor allem der Bau und die Eröffnung der Unterkunftshütte Hinterbärenbad am 29. Juli 1883 durch die Sektion Kufstein des DuOeAV ein wichtiger Schritt. ${ }^{63}$ Es handelte sich um die erste Alpenvereinshütte im Kaisergebirge, die rein auf alpine Gäste ausgerichtet war. Davor standen den Bergsteiger*innen nur die bestehenden Almhütten als Unterkunft zur Verfügung, wo sie gegen Entgelt von den Senner*innen untergebracht und aufWunsch verpflegt wurden. Die Hüttenwirtsleute von Hinterbärenbad, das Ehepaar Nani und Paul Horngacher, versorgten die wandernden und kletternden Gäste und waren nebenbei auch als Gelegenheitsbergführer*innen tätig. Bereits 1884 wurden 400 Besucher*innen auf der Hütte gezählt und aufgrund der anhaltenden Bergbegeisterung und des Zulaufs von Vereinsangehörigen in den diversen Sektionen des DuOeAV nahmen die Gästezahlen der Hütte von Jahr zu Jahr zu, sodass 1890 bereits 1620 Bergsteiger*innen verzeichnet wurden. ${ }^{64}$ Der weitere Ausbau sowie die Verbesserung der Wege und Steige sorgten ebenso für einen regen Zulauf im Kaisergebirge. Schon vor der Eröffnung der Unterkunftshütte wurde ein Weg von Hinterbärenbad zur Pyramidenspitze gebaut. In den Jahren nach der Eröffnung kamen weitere Wege im Gebiet dazu und sorgten für eine weitere Attraktivitätssteigerung. ${ }^{65}$ Ein zusätzlicher Aspekt mag die rasche und unkomplizierte Erreichbarkeit der Hütte gewesen sein, da die Wanderung von Kufstein nach Hinterbärenbad auch für Ungeübte leicht zu bewältigen war.

Die bereits angeführte Steigerung der Gästezahl führte rasch zu einem Kapazitätsproblem in der Unterkunftshütte und so wurde der Plan zum Bau eines neuartigen Alpengasthauses, dem Anton-Karghaus Hinterbärenbad, gefasst. Dieses moderne Domizil, das mit einem Badehaus und einer Telefonverbindung ausgestattet war, wurde 1896 eröffnet. Nachdem es 1899 bei einem verheerenden Brand komplett zerstört wurde, machte sich die Alpenvereinssektion daran, das Haus rasch wiederaufzubauen und im folgenden Jahr wieder zu eröffnen. Hinterbärenbad verfügte nun über neunzig Betten in 42 Zimmern und zwölf Matratzenlager, eine eigene Hausapotheke und sogar eine Dunkelkammer für die Hobbyfotografie. In den Sommermonaten war die Hütte oftmals so ausgelastet, dass Gäste im Maultier-Stall übernachten mussten. Entsprechend groß war die Anzahl des Personals auf der Hütte. Auf einer Fotografie aus dem Jahre 1908 sind 26 Personen abgebildet, davon waren sieben Personen Teil der Pächterfamilie, die restlichen waren Angestellte. ${ }^{66}$

In einem Heft, welches von der Alpenvereinssektion vermutlich in der Zeit nach 1904 herausgegeben wurde, wird deutlich, welchen Stellenwert Hinterbärenbad und das Kaisergebirge hatten. Neben einer Beschreibung der Einrichtung des Unterkunftshauses, das als eines der „besteingerichteten Schutzhäuser der Ostalpen“ galt, wurden die Touren ab Hinterbärenbad erläutert. Allein die Überschrift „Hauptausgangspunkt für alle

63 Deutsche und Österreichische Alpenvereinssektion Kufstein (Hrsg.), Die Erschließung des Kaisergebirges. Nach Aufzeichnungen in Hinterbärenbads Tourenbüchern bearbeitet von F. Nieberl, Kufstein 1912² S. 12.

64 Bendler, Wilder Kaiser, S. 123.

65 Bendler hat die wichtigsten Wegerschließungen im Kaisergebirge sehr gut zusammengefasst. Ebd., S. 117-118.

66 Deutsche und Österreichische Alpenvereinssektion Kufstein (Hrsg.), Die Erschließung des Kaisergebirges. Nach ebd., S. 123-124. 
Kaisergebirgstouren" zeigt, dass es für jeden bergbegeisterten Gast in Kufstein fast unumgänglich war, Hinterbärenbad zu besuchen. ${ }^{67}$

Das Kaisergebirge wurde durch die Erschließung der Alpenvereinssektion nicht nur für Bergsteiger*innen interessant, sondern auch für den bequem wandernden Gast. Die Wanderungen im Kaisergebirge wurden neben Spaziergängen zum zentralen Bestandteil des Ferienprogramms der Sommergäste. Das zeigt sich in den Tourenbeschreibungen des oben genannten Heftes genauso wie im Fremdenführer aus dem Jahre 1904, in welchem das Kaisergebirge mit seinen Touren ausführlich beschrieben wurde. ${ }^{68}$ Auch im Baedecker Handbuch aus dem Jahre 1912 wurde in der Überschrift des Beitrags „Kufstein und das Kaisergebirge" deutlich, dass aus Sicht des Fremdenverkehrs die Stadt und das Gebirge eine untrennbare Einheit darstellten. ${ }^{69}$ Das Hausprospekt des Gasthofs zum Auracher zeigt ebenfalls die Bedeutung des Kaisergebirges:

„Die eigentliche Schatzkammer der Natur Kufsteins ist der,Kaiser', das charakteristischste Gebirge des Unterlandes, überreich an landschaftlichen Schönheiten und an interessanten Sagen. Ein ganz besonderer Zauber ist dem ,Kaiser' eigen: Dem Ankömmling aus dem nahen Flachlande, z. B. aus der benachbarten Großstadt München, eröffnet er schon nach kurzer, müheloser Wanderung den Einblick in die großartigste Gebirgswelt, wie man sie herrlicher und überwältigender auch im Innern des Landes nicht umher schauen kann. Man gelangt dahin an dem romantisch-idyllischen Sparchen und an der Geisterschmied-Wand vorüber in das berühmte Kaisertal. Erquickender Duft von Nadelholzbäumen, Wasserrauschen, Fels- und Steingeröll, dann wieder hübsche Oasen und Bauernhöfe und das Hinterbärenbad empfängt uns mit lauterem Wohlwollen, die hohen steilen Felszacken winken uns den Willkommensgruß zu." ${ }^{\prime \prime 0}$

Über die anderen Sektionen des DuOeAV und über die Publikationen und Karten der Sektion Kufstein wurde das Kaisergebirge weiter bekannt gemacht. Somit kann der Alpenverein fast als eine Art alpiner Tourismusverband betrachtet werden, der maßgeblich zur Stärkung des Fremdenverkehrs im Sommer beitrug.

\section{Kufstein als bedeutendes Fremdenverkehrsziel im Sommer}

In den Folgejahren wurden weitere Schritte gesetzt, die das touristische Angebot in Kufstein kontinuierlich verbesserten. 1891 kam es zur Gründung des Vereins zur Hebung des Fremdenverkehrs, dessen Vereinszweck noch stärker auf die Förderung des Fremdenverkehrs ausgerichtet war, als dies beim Verschönerungsverein der Fall war. Erster Vorstand dieses Vereins wurde der damalige Bürgermeister Hans Reisch. Dekan Hörfarter war als Ausschussmitglied ebenfalls beteiligt. Da der Verein schon im Gründungsjahr

67 Sektion Kufstein des Deutschen und Österreichischen Alpenvereins (Hrsg.), Hinterbärenbad im Kaiser-Gebirge. Ein Geleit- und Erinnerungs-Büchlein für Kaisergebirgs-Freunde, Kufstein 1904, S. 4.

68 Verein zur Hebung des Fremdenverkehrs, Kufstein, S. 103.

69 Karl Baedeker, Südbayern, Tirol, Salzburg, Ober- und Nieder-Österreich, Steiermark, Kärnten und Krain. Handbuch für Reisende, Leipzig 1912 ${ }^{35}$, S. 211.

70 Prospekt Gasthof zum „Auracher", S. 1. 
über fünfzig Mitglieder verfügte, zeigt sich, dass die Förderung des Fremdenverkehrs für die Stadt bereits 1891 bedeutend war. ${ }^{71}$ Der Verschönerungsverein war es auch, welcher den bereits mehrfach erwähnten Reiseführer 1904 herausgab. Weiters wurde ab 1895 die Zahl der Fremden in den Sommermonaten in einer Fremdenliste dokumentiert. Gleichzeitig diente dieses Medium mit verschiedenen Inseraten als Werbung.

Neben diesem neuen Verein kam es in Kufstein zu Verbesserungen in der touristischen Infrastruktur. Bestehende Gasthäuser und Hotels passten ihr Angebot an die Erwartungen der Gäste an und moderne Neubauten wurden errichtet. 1906 eröffnete der Kufsteiner Getreidehändler Josef Dillersberger das Hotel Zu den 3 Königen. Diesem Bau folgte dann 1909 die Neueröffnung des Hotels Egger mit angeschlossener Brauerei am Oberen Stadtplatz, welches dem von 1904 bis 1919 amtierenden Bürgermeister Josef Egger gehörte. ${ }^{72}$ Die beiden neuen Hotels mitten im Stadtzentrum rundeten das bereits bestehende Angebot an Unterkünften ab. Kufstein konnte gerade durch diese Neubauten bei den Gästen mit modernen Annehmlichkeiten punkten. Das Hotel Egger beispielsweise bot seinen Gästen einen Fahrstuhl und komfortable Zimmer mit hochwertiger Einrichtung, welche großteils aus Wien stammte. Es verfügte zudem über Zimmer mit eigenem Salon, Bad und Toilette. Besonders stolz schienen die "elektrisch betriebenen Vacuumreiniger" zu machen, die für ein staubfreies Hotel sorgten.73

Die fortschrittliche Wasserversorgung und die rasche Elektrifizierung der Stadt hatten ebenfalls positive Auswirkungen auf den Fremdenverkehr. Im Herbst 1894 wurde eine neue Wasserleitung aus dem Kaisertal in Betrieb genommen, die nicht nur die zwanzig öffentlichen Brunnen, sondern auch zweihundert private Objekte sowie die umliegenden Weiler mit Wasser versorgte. Bis 1914 war die Wasserversorgung mit dem Hochquellenwasser aus dem Kaisertal so angelegt, dass mindestens 10.000 Menschen mit zweihundert Liter Wasser pro Tag versorgt werden hätten können. Neben der Wasserversorgung war auch die Elektrifizierung, welche 1897/1898 mit der Errichtung des Elektrizitätswerks einsetzte, fortschrittlich und trug zum Ruf Kufsteins als moderner Fremdenverkehrsort bei. Beides waren wichtige Komfortaspekte, welche die steigende Nachfrage nach einem Aufenthalt in Kufstein verstärkten. ${ }^{74}$

Alle in Kufstein gesetzten Maßnahmen führten dazu, dass die Gästezahl stetig wuchs und der Sommertourismus wirtschaftlich bedeutend wurde. Die folgenden Zahlenwerte illustrieren diese Entwicklung des Fremdenverkehrs hin zu einem wichtigen Wirtschaftsfaktor. Ein wichtiges Merkmal ist die Anzahl an angebotenen Fremdenbetten, dazu findet sich im Reiseführer aus dem Jahr 1904 ein Hinweis: „Im Jahre 1903 besuchten Kufstein 13000 Fremde, welche zusammen 56000 mal übernachteten, was bei einer Bettenzahl von 1060 eine durchschnittliche Bettbenützung von 53 im Jahre ergibt." ${ }^{175}$ 1.060 Fremdenbetten standen demnach 1903 in Kufstein zur Verfügung. Davon waren

71 Blattl, Fremdenverkehr, S. 18-19.

72 Franz Biasi, Kufstein. 600 Jahre Stadt 1393-1993, Innsbruck-Wien 1992, S. 195.

73 Prospekt Hotel Egger (Familie Greiner), o. D. (nach 1909). Hannover-Ricklingen,S. 7-8. Es handelt sich um das erste und größte Familien- und Touristenhaus am Platz.

74 Biasi, Kufstein, S. 188-190.

75 Verein zur Hebung des Fremdenverkehrs, Kufstein, S. 58. 
der Großteil gewerbliche Betten in Gasthöfen und Hotels, denn laut der vom Fremdenverkehrsverein publizierten Wohnungsliste der Sommersaison 1901 wurden in den Privatwohnungen insgesamt 150 Betten angeboten. ${ }^{76}$ Die Relation der Gästebetten zur Einwohnerzahl zeigt ebenfalls die bereits Anfang des 20. Jahrhunderts wirtschaftliche Wichtigkeit des Fremdenverkehrs. Bei der Volkszählung im Dezember 1900 wurden in Kufstein und seinen Vororten 4.369 Einwohner*innen gezählt.77 Auf jede vierte Person kam somit ein Gästebett.

Auch die Entwicklung der Gästezahl gibt einen Hinweis darauf, dass der Fremdenverkehr zum Wirtschaftsfaktor wurde. In der "Kufsteiner Chronik“ findet sich im Jahr 1904 ein Eintrag zur Generalversammlung des Fremdenverkehrsvereins: Von 1892 bis 1903 hatte sich die Gästezahl in Kufstein mehr als verdoppelt. Während 1892 noch 5.922 Gäste und 25.415 Übernachtungen verzeichnet wurden, waren es 1903 bereits 13.087 Gäste und 56.274 Übernachtungen. ${ }^{78}$ Hierbei dürfte es sich um die absoluten Zahlen der beiden Jahre handeln, denn in den von 1895 bis 1910 regelmäßig erschienenen Fremden- und Wohnungslisten wurden alle Gäste in den Sommermonaten von Anfang Juli bis Mitte September statistisch erfasst, die länger als drei Nächte in Kufstein verweilten. Auch wenn es sich hier um eine selektive Auswahl der Gästezahl aufgrund der Aufenthaltsmonate handelt, so belegen sie einen grundsätzlich stetigen Zuwachs an Sommergästen in Kufstein in diesen 15 Jahren.

Beim Vergleich der Gästezahlen aus der obenstehenden Abbildung mit den vorhin erwähnten Zahlen aus der Generalversammlung 1904 ist eine Abweichung für das Jahr 1903 erkennbar. Diese Differenz lag vermutlich an den Gästen, die außerhalb der in den Fremdenlisten erfassten Sommermonate in Kufstein verweilten.

\begin{tabular}{|l|l|l|l|}
\hline Jahr & $\begin{array}{l}\text { Gäste- } \\
\text { zahl }\end{array}$ & Jahr & $\begin{array}{l}\text { Gäste- } \\
\text { zahl }\end{array}$ \\
\hline 1895 & 6.743 & 1903 & 10.063 \\
\hline 1896 & 5.504 & 1904 & 10.738 \\
\hline 1897 & 5.816 & 1905 & 10.198 \\
\hline 1898 & 7.037 & 1906 & 10.596 \\
\hline 1899 & 8.834 & 1907 & 11.878 \\
\hline 1900 & 7.804 & 1908 & 11.763 \\
\hline 1901 & 8.005 & 1909 & 11.919 \\
\hline 1902 & 9.877 & 1910 & 14.439 \\
\hline
\end{tabular}

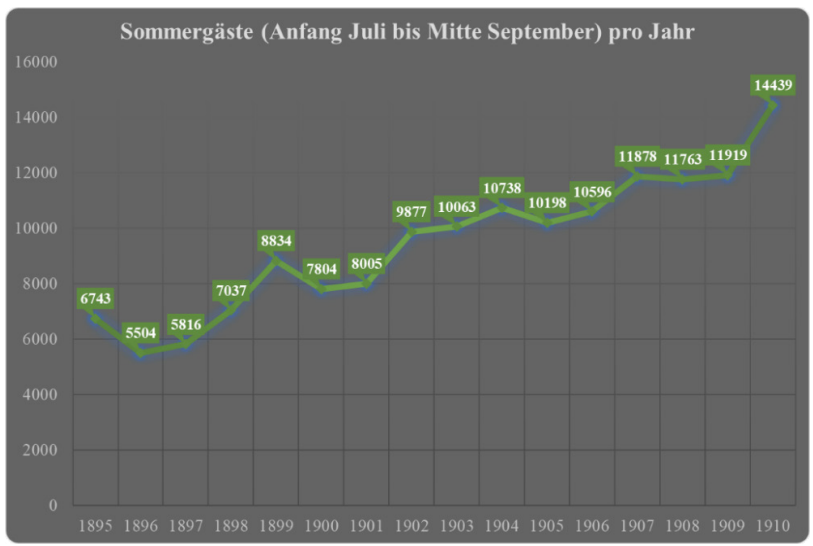

Abbildung 1: Die Entwicklung der Sommergäste-Anzahl von 1895 bis 1910 - Eigene Darstellung auf Basis der Daten aus den Fremden- und Wohnungslisten der Jahre 1895 bis $1910 .^{79}$

76 Lippott, Chronik, S. 75

77 Ebd., S. 74-75.

78 Ebd., S. 82-83.

79 Verein zur Hebung des Fremdenverkehrs in Kufstein (Hrsg.), Fremden- \& Wohnungsliste für Kufstein und das Kaisertal, Kufstein 1895-1904; Fremdenverkehrs-Verein Kufstein (Hrsg.), Kufsteiner Fremden-Liste, Kufstein 19051910. 


\section{Der Wintertourismus in Kufstein}

Nachdem Kufstein in der Sommersaison bereits erste Erfolge und wirtschaftliche Vorteile erzielen konnte, bemühte sich die Stadt Anfang des 20. Jahrhunderts um die Erschließung der Wintersaison. Bei der Generalversammlung des Fremdenverkehrsvereins wurde 1904 entsprechend festgehalten: Es wird angestrebt, den Fremdenverkehr auch im Winter zu heben." 80 Wichtig und zentral für den wirtschaftlich relevanten Ausbau der touristischen Wintersaison war, dass auf eine funktionierende touristische Vermarktung und auf ein bereits erprobtes Beherbergungs- und Gaststättenwesen zurückgegriffen werden konnte, das ebenfalls an einer Ausweitung der Saison interessiert war.

\subsection{Wann begann die Wintersaison in Kufstein?}

Die in dieser Arbeit verwendeten Quellen geben Hinweise darauf, dass sich die Wintersaison in Kufstein zu Beginn des 20. Jahrhunderts entwickelte und mit dem Wintersport zusammenhing: So ist im Reiseführer von 1904 zu lesen, dass der Aufschwung des Wintersports in den letzten Jahren spürbar war. ${ }^{81}$ Auch das Baedecker Handbuch für Reisende aus dem Jahr 1912 zeigt, dass Kufstein bereits zu diesem Zeitpunkt Winterreiseziel war: „Kufstein (503m), Stadt mit 6500 Einw., als Sommerfrische und Wintersportplatz viel besucht, liegt am r. Ufer des Inn, [...]." ${ }^{\prime \prime 2}$ Weiters findet sich folgender Hinweis: „Viele Kurorte und Gasthöfe, die früher im Herbst schlossen, haben jetzt eine belebte Wintersaison." ${ }^{\prime 83}$ Die Gründe für diese Entwicklung lagen einerseits im Gesundheitsaspekt, der sich vor allem in der frischen, trockenen und sauberen Winterluft zeigte, andererseits im Angebot an Wintersportmöglichkeiten. ${ }^{84}$

Im Prospekt des Hotels Egger ${ }^{85}$ wird explizit um den Wintergast geworben und auf einige Annehmlichkeiten hingewiesen:

"Als Wintersportplatz hat Kufstein bedeutenden Aufschwung genommen, denn durch die Unterstützung seitens der k.k. Staatsregierung wurde es möglich, eine sportlich wie landschaftlich schöne, 3,2 Kilometer lange Bobsleighbahn herzustellen. Des weiteren befinden sich in der nächsten und weiteren Umgebung vier Rodelbahnen, eine Schlittschuhbahn und ein sowohl für Anfänger als für Geübte geeignetes Skigelände. Mit Rücksicht auf den Wintersport wurde im Haus Niederdruckdampfheizung eingerichtet, die alle Räume und Gänge gleichmäßig angenehm durchwärmt, Auch Trockenräume für nasse Kleider sind vorgesehen, [...]. An Sportslustige werden auch Geräte aller Art, Bobs, Sceletons, Rodelschlitten, Skier, leihweise abgegeben." ${ }^{\text {"6 }}$

\footnotetext{
80 Lippott, Chronik, S. 83.

81 Verein zur Hebung des Fremdenverkehrs, Kufstein, S. 58-59.

82 Baedeker, Handbuch, S. 211.

83 Ebd., S. 18

84 Ebd.

85 Das Prospekt ist undatiert, entstand aber vermutlich nach dem Neubau des Hotels 1909 und vor Beginn des Ersten Weltkrieges 1914

86 Prospekt Hotel Egger, S. 8-9.
} 
Laut diesem Hotelprospekt gab es aufgrund des Wintersports fast keinen Unterschied hinsichtlich der Gästeanzahl im Sommer und Winter: „An manchem Sonntag in den kalten Monaten sieht der Bahnhof Kufstein ebenso viele Gäste wie in der Hochflut der Reisezeit. " ${ }^{77}$ Natürlich sind Werbeprospekte kritisch zu betrachten, weil diese primär den Wünschen und Bedürfnissen der Gäste entsprechen wollen. Die Beschreibung des 1909 errichteten Hotels zeigt allerdings, dass der Wintergast beim Neubau damals bereits berücksichtigt wurde. Die Wintersaison musste also in den Jahren vor 1909 begonnen haben, sonst hätte das Hotel vermutlich auf die Investition in eine wintertaugliche Ausstattung verzichtet.

Neben dem Fremdenverkehrsverein unterstützte die 1904 durch Hans Reisch gegründete Wintersportvereinigung die Wintersaison in Kufstein. Mit diesem neuen Verein begann der Auf- und Ausbau der Wintersportstätten wie Bob- und Rodelbahnen, Sprungschanzen und Skipisten. ${ }^{88}$ Kufstein schuf so eine Infrastruktur für den Wintersport, ohne die eine Wintersaison wohl nicht denkbar gewesen wäre.

Auch das Winterprospekt des Fremdenverkehrsvereins ${ }^{89}$ verweist darauf, dass sich Kufstein in den zurückliegenden Jahren dem Wintertourismus zugewandt hatte:

„Schüchtern erst, dann mit etwas mehr Mut, hat Kufstein die letzten paar Winter in der Welt draußen laut werden lassen, wie schön es ist in seinen winterlichen Tälern, auf seinen Höhen in strahlendem Sonnenschein, in der traulich warmen Stube des eingeschneiten Bauernhauses, in den eleganten Räumen der vornehmen Hotels! Und der Erfolg blieb nicht aus. Sportsleute und Winterfreunde aller Art sind gekommen, und gefallen hat's ihnen allen.."90

\subsection{Die enge Verbindung von Wintersaison und Wintersport}

Die Entwicklung hin zu einer Wintersaison ist untrennbar mit dem Wintersport verbunden. Vermutlich nach skandinavischem Vorbild fanden Schneeteller und Skier Einzug in Tirol. Somit konnte der populär gewordene Bergsport auch im Winter fortgeführt werden. Bergführer*innen wagten sich gegen Ende des 19. Jahrhunderts in Winterbesteigungen auf die Tiroler Gipfel. Diesem neuen Trend verschloss sich auch der Alpenverein nicht und veranstaltete erste Skikurse für Bergführer*innen. Die neu gegründeten Wintersportvereinigungen und Skiclubs verschrieben sich ganz dem Wintersport. Die ersten Vereine in Nordtirol waren der 1901 gegründete Skiclub Arlberg sowie der 1902 gegründete Wintersportverein Kitzbühel. Kufstein folgte, wie bereits erwähnt, 1904 diesen beiden Beispielen. ${ }^{91}$

Bald nach dem Aufkommen des Skisports in Tirol wurden 1906 die ersten Skiführer für das Gebiet herausgegeben. Neben diesen Büchern trug die Austragung von Skirennen

87 Ebd., S. 2.

88 Eduard Lippott (Hrsg.), 60 Jahre Tiroler Grenzbote 1871-1931, Kufstein 1931, S. 77.

89 Das Prospekt wurde zwar ohne Jahreszahl herausgegeben, der Inhalt lässt aber auf eine Veröffentlichung für die Wintersaison 1911/12 schließen.

90 Wintersportvereinigung Kufstein (Hrsg.), Winter in Kufstein, München 1911, S.3.

91 Lässer, Fremdenverkehr, S. 86-88. 
ebenfalls zur Popularität des Skisports bei. Auch das Skispringen gehörte zum Skisport, weshalb in den Wintersportzentren vor dem Ersten Weltkrieg Sprungschanzen errichtet wurden. ${ }^{92}$

In Kufstein wurde der Ski- und Wintersport vom Fremdenverkehrsverein, von der Sektion Kufstein des DuOeAV und von der Wintersportvereinigung gefördert. Diese Institutionen erhielten mit dem Kufsteiner Skiclub, der 1910 gegründet wurde, einen wichtigen Partner. ${ }^{93}$ Zusätzlich förderten in Kufstein die Stadt und die k. k. Staatsregierung den Wintersport und damit die Wintersaison. ${ }^{94}$ Die Bemühungen um den Winter schienen Erfolg zu haben: Kufstein konnte sich als frühes Wintersportzentrum etablieren. Dies dokumentieren nicht nur die Kufsteiner Quellen, sondern auch das Reisehandbuch von Karl Baedecker, das Kufstein 1912 unter den ausgewählten und als „besonders bedeutend" markierten Wintersportorten in Tirol und Vorarlberg führt,95 sowie der "Fremdenverkehrs- und Sports-Anzeiger in Tirol, Vorarlberg und Liechtenstein“ aus dem Jahr 1909. In diesem wird Kufstein als einer von insgesamt 14 möglichen Wintersportplätzen in Tirol und Vorarlberg angeführt. Gerade in den erfolgreichen Orten gab es ein breites Angebot, das neben dem Skilauf und dem Skispringen auch Eislaufen und verschiedene Arten des Rodelsports umfasste. Kufstein zählte der Zeitschrift zufolge zu den fünf angeführten Orten, die mindestens fünf Wintersportmöglichkeiten anboten. Nur Innsbruck und Kitzbühel boten mehr Sportmöglichkeiten. Für Kufstein werden Eislaufen, Eisschießen, Ski, Bobsleigh und Rodeln als Sportarten angegeben, das gleiche Angebot hatte St. Anton am Arlberg. ${ }^{96}$ Die Tatsache, dass Kufstein in diese Tabelle aufgenommen wurde, zeigt die Bedeutung der Stadt als Wintersportort vor dem Ersten Weltkrieg.

Inwiefern Kufstein von der auf Tiroler Ebene durchgeführten Werbetätigkeit profitiert hat, ist nicht direkt nachvollziehbar. Jedoch trugen die Bemühungen rund um die Wintersaison vom 1890 gegründeten Landesverband für Fremdenverkehr sicherlich ihren Teil dazu bei, dass Gäste Tirol vermehrt als Winterdestination entdeckten. Interessant ist in diesem Zusammenhang, dass Parallelen zu Kufstein bestehen. So erfolgten die ersten Werbemaßnahmen zur selben Zeit, als in Kufstein der Wintersport 1904/1905 institutionalisiert wurde. Vom Landesverband wurde bereits für die Wintersaison 1904/1905 ein Plakat zum Thema Wintersport in Tirol herausgegeben. 1910 folgten jeweils eine deutsche und eine englische Broschüre, die sich dem Thema Winterurlaub in Tirol widmeten. ${ }^{97}$ Möglicherweise dienten diese Werbemaßnahmen als Vorbild für das bereits mehrfach erwähnte Kufsteiner Winterprospekt.

92 Michael Forcher, Zu Gast im Herzen der Alpen. Eine Bildgeschichte des Tourismus in Tirol, Innsbruck 1989, S. 154156.

93 Herbert Kuntscher, Wintersport seinerzeit, in: Hans Treichl (Hrsg.), Kufstein. Bayerns Glanzperle Tirols, Kufstein 2012, S. 168-175, hier S. 169.

94 Wintersportvereinigung, Winter, S. 11.

95 Baedeker, Handbuch, S. 14.

96 Fremdenverkehrs-und Sports-Anzeiger in Tirol, Vorarlberg-Liechtenstein (1909), Heft 1, zit. n. Michael Forcher, Zu Gast im Herzen der Alpen. Eine Bildgeschichte des Tourismus in Tirol, Innsbruck 1989, S. 165. 


\subsection{Wintersport in Kufstein}

Kufsteins Vorteil im Bemühen um eine Wintersaison lag vor allem im breiten Angebot an Wintersportmöglichkeiten. Die Stadt setzte einen Schwerpunkt im Rodelsport, aber auch Skifahren, Skispringen und Eislaufen waren Aktivitäten, die in der Kufsteiner Wintersaison möglich waren.

Eine besondere Rolle in der Kufsteiner Wintersaison spielte die Wintersportvereinigung, deren Vereinszweck dem Ausbau und der Instandhaltung der Wintersportanlagen gewidmet war. Im eigenen Winterprospekt stellte sich der Verein als sehr eifrig dar:

„Die seit dem Jahre 1904 bestehende rührige Wintersportvereinigung hat es sich, unterstützt durch Stadt und Regierung, angelegen sein lassen, eine ganze Reihe mustergültig angelegter Sportsbahnen und -plätze zu schaffen und für deren bestmögliche Unterhaltung Sorge zu tragen. Rodel- und Skisport, Bobsleigh und Skeleton, Eislauf und Schlittenfahrt finden infolgedessen liebevolle Pflege und eifrige Ausübung." ${ }^{198}$

Es bestand wohl eine wechselseitige Beziehung zwischen der Wintersportvereinigung und dem Fremdenverkehrsverein: Einerseits unterstützte die Wintersportvereinigung das 1904 vom Fremdenverkehrsverein gesetzte Ziel, eine zweite Saison aufzubauen und war in dieser Arbeit fleißig um neue Wintersportmöglichkeiten bemüht. Andererseits wäre vermutlich eine Gründung der Wintersportvereinigung gar nicht erst zustande gekommen, wenn es nicht bereits die Vision einer Wintersaison gegeben hätte.

\subsection{Rodeln und Bobsleigh}

Kufstein kann als frühes Rodelmekka bezeichnet werden. Im Winterprospekt wird das Thema Rodeln und Bobsleigh ausführlich behandelt und nimmt im 17-seitigen Prospekt ganze vier Seiten ein. Zum Vergleich: Dem Skisport wurden zweieinhalb Seiten gewidmet.99

Das Prospekt "Winter in Kufstein“ setzte innerhalb der Rodelinformationen einen Schwerpunkt auf die Bobsleighbahn. Diese Bahn wurde sehr detailliert beschrieben und dürfte damit wohl einen besonders hohen Stellenwert gehabt haben. Die Erbauung der 3.200 m langen Bahn wurde von der k. k. Staatsregierung subventioniert. Der Start der Bobsleighbahn konnte über einen eigens angelegten Weg, auf dem auch die Zugtiere die Bobs transportierten, erreicht werden. Zwischen dem Start und dem Ziel war eine Kommunikation mittels Telefon möglich. ${ }^{100}$ Die Bahn wurde Ende Dezember 1908 mit einem Rennen eröffnet. ${ }^{101}$ Die anderen der insgesamt sieben Rodelbahnen wurden im Prospekt ebenso mit zahlreichen Details beschrieben. Angeführt wurden

\footnotetext{
98 Wintersportvereinigung, Winter, S. 9.

99 Ebd., S. 11-13.

100 Ebd.

101 Lippott, Chronik, S. 104.
} 
etwa die Schwierigkeit und die Länge der Bahn sowie eine Beschreibung der Aussicht während der Fahrt. ${ }^{102}$

Einige Kufsteiner Unterkünfte, wie etwa das Hotel Egger, stellten ihren Wintergästen Rodel, Bobs und Skeletons leihweise zur Verfügung. ${ }^{103}$ Waren keine Sportgeräte im Hotel ausleihbar, verfügten die an den Rodelbahnen gelegenen Gasthöfe über Leihrodel. ${ }^{104}$

\subsection{Skispringen \& Skifahren}

Skifahren und Skispringen gingen in dieser frühen Zeit des Wintertourismus noch Hand in Hand, meist wurden Sprungschanzen am jeweiligen Skigelände angelegt. Anfänger*innen standen in Kufstein Übungsplätze auf den Hügeln der Stadt zur Verfügung, beispielsweise im Ortsteil Zell am Lausbichl oder am Thierberg. Auch im Bereich rund um Vorderdux und Hinterdux konnten Gäste und Einheimische erste Skierfahrungen sammeln. Zusätzlich gab es die Möglichkeit, die von der Wintersportvereinigung von Mitte Dezember bis Mitte März regelmäßig angebotenen Skikurse zu besuchen. ${ }^{105}$ Nach der Gründung des Kufsteiner Skiclubs 1910 widmete sich dieser ebenso der Durchführung von Skikursen: „Der Aufgabenkreis der Skiclubs umfaßte Tourenführungen, Verbesserungen der Technik durch Schulung und Veranstaltungen von Rennen." ${ }^{106}$

Das bereits erwähnte Gebiet rund um Vorder- und Hinterdux war im Grunde das Kufsteiner Wintersportzentrum. Rodeln, Bob- und Skeletonfahren, Skispringen und Skirennen waren dort möglich. Das nahegelegene Almengebiet um das Brentenjoch und den Steinberg ergänzte mit den Skipisten das Angebot. Vor allem die Schneelage, die gute Lage der Abfahrten aufgrund der weitläufigen Almwiesen sowie die abwechslungsreichen Möglichkeiten dieses Bereichs wurden im Winterprospekt hervorgehoben: Dieses Gebiet würde das „Prädikat,ideal“" verdienen, denn es „weist alles auf, was des Skiläufers Herz höher schlagen lässt". ${ }^{107}$ Laut Franz Biasi rühmte sogar eine Frankfurter Zeitung den Skisportplatz Steinberg als „Paradies für Skifahrer".108

Kufstein schien in der Wintersaison damit zu kämpfen, dass das im Sommer so wichtige Kaisertal mit Hinterbärenbad für Skifahrer*innen als nicht ideal wahrgenommen wurde und für die Bewerbung nicht ausreichend war. Um die Skigäste anzusprechen, musste in der Argumentation also auf andere Regionen, wie den Steinberg und Vorder- und Hinterdux, gesetzt werden:

„Es ist eine leider noch immer vielfach verbreitete Ansicht, dass Kufstein und insbesondere der Kaiser, so gar kein Skigelände' besäßen. Diese Meinung teilen merkwürdigerweise auch viele, die Kufstein und den Kaiser sehr gut zu kennen meinen. Gewiß - Hinterbärenbad, das für so viele identisch ist mit dem ,Kaiser',

\footnotetext{
102 Wintersportvereinigung, Winter, S. 11-13.

103 Prospekt Hotel Egger, S. 9.

104 Wintersportvereinigung, Winter, S. 12-13.

105 Ebd., S. 13-15.

106 Lässer, Fremdenverkehr, S. 89.

107 Wintersportvereinigung, Winter, S. 15.

108 Biasi, Kufstein, S. 201.
} 
darf kaum für sich das Prädikat, Standquartier für Skiläufer' in Anspruch nehmen, wenn auch der bescheidenere Fahrer dort schon ganz gut auf seine Rechnung kommt. Aber es gibt noch andere Orte als Hinterbärenbad und Stripsenjoch, als Halt und Totenkirchl, die auch zum ,Kaiser' gehören und die selbst für den verwöhnten Skimann noch eine ganze Menge Leckerbissen aufbewahren."109

Mit Vorderdux, Hinterdux, Steinberg und den Übungshügeln gelang es Kufstein, sich als Skiort zu etablieren und damit auch skifahrende Gäste anzulocken.

\subsection{Die Wintersportfeste - Werbung für Kufsteins Wintersaison}

Eine kluge „Erfindung" der Wintersportvereinigung mit anhaltender Wirkung für die beginnende Wintersaison waren die Wintersportfeste. Das erste Wintersportfest fand vom 21. bis 23. Januar 1905, ein Jahr nach Gründung der Wintersportvereinigung, statt und wurde danach regelmäßig einmal pro Winter veranstaltet. ${ }^{110}$ Die Teilnehmer*innen der Wintersportfeste bekamen einen Einblick in die Vielfalt der Wintersportmöglichkeiten: Das Programm umfasste Eisschießen, Rodelrennen für Damen und Herren sowie Skiwettkämpfe mit Skirennen und Skispringen und ein gesellschaftliches Rahmenprogramm, u. a. die musikalische Umrahmung durch die Musikkapelle. Die Presse berichtete über die Kufsteiner Wettbewerbe im deutschsprachigen Raum, also dem heutigen Deutschland, in der Schweiz und in Österreich. ${ }^{111}$

Für das zweite Wintersportfest 1906 belegen die Zahlen der „Kufsteiner Chronik" bereits einen regen Zulauf. Alleine aus dem Münchner Raum kamen 1500 Gäste in zwei Sonderzügen nach Kufstein. Der Zuwachs dürfte angehalten haben, 1907 sind 2000 Gäste aus dem Münchner Raum als Teilnehmer*innen des Wintersportfestes erwähnt..12

Die Wintersportvereinigung schien sehr bemüht gewesen zu sein und sorgte immer wieder für Neuheiten. So wurde beispielsweise ein Paar-Rodel-Bewerb abgehalten oder auch das Skijöring mit einem Pferdegespann eingeführt. Die Anlage eines neuen Eislaufplatzes am Fischergries 1908 mit einer Fläche von 2.000 Quadratmetern steht ebenfalls in Zusammenhang mit den Wintersportfesten. Die Wintersportfeste trugen ihren Teil dazu bei, dass Kufstein seinen Ruf als Wintersportort festigen konnte und der Wintertourismus in Kufstein zu einem Wirtschaftsfaktor werden konnte. ${ }^{113}$

\subsection{Zur Größenordnung des frühen Wintertourismus}

Durch den Vergleich der statistischen Daten aus verschiedenen Quellen sind Abweichungen erkennbar, die aus Sicht der Verfasserin Hinweise auf die Größenordnung der Saison außerhalb des Sommers in Kufstein liefern können. Die Gästezahlen aus den Fremden- und Wohnungslisten bezogen sich rein auf die Sommersaison von Anfang

\footnotetext{
109 Wintersportvereinigung, Winter, S. 14.

110 Lippott, Chronik, S. 85.

111 Biasi, Kufstein, S. 200-201.

112 Lippott, Chronik, S. 85.

113 Biasi, Kufstein, S. 201.
} 
Juli bis Mitte September. Für einige der in Abbildung 1 dargestellten Jahre lagen noch andere Zahlen vor, die in der folgenden Abbildung dargestellt sind und anhand derer ein Vergleich mit den statistischen Daten aus der Sommersaison vorgenommen werden kann.

\begin{tabular}{|l|l|l|l|}
\hline Jahr & Sommergäste ${ }^{114}$ & Gäste gesamt & Gästedifferenz \\
\hline 1903 & 10.063 & $13.000^{115}$ & 2.937 \\
\hline 1909 & 11.919 & $18.266^{116}$ & 6.347 \\
\hline 1910 & 14.439 & 26.624 & 12.185 \\
\hline
\end{tabular}

Abbildung 2: Gästeanzahl in Kufstein außerhalb der Sommerhochsaison (eigene Darstellung) ${ }^{17}$

Während 1903 - also vor Einsetzen der relevanten Wintersaison - im Verhältnis noch eine sehr geringe Anzahl an Gästen außerhalb der Sommersaison für Kufstein gezählt wurde, stellte sich die Situation für die Jahre 1909 und 1910 anders dar. 1909 kamen zwei Drittel der Gesamtgäste in der Sommersaison nach Kufstein, 1910 waren es mehr als die Hälfte aller Gäste. Es ist in diesen beiden Jahren anhand der Gästedifferenz jedoch die Tendenz erkennbar, dass auch außerhalb der Sommermonate eine beachtliche Anzahl an Gästen Kufstein besuchte. Wird diese Gästedifferenz nun in Relation zu den vorangegangenen Schilderungen über die Wintersaison gesetzt, so kann angenommen werden, dass die außerhalb der Sommersaison gezählten Gäste zu einem wesentlichen Teil in der Wintersaison nach Kufstein kamen. Die These, dass die Wintersaison vor dem Ersten Weltkrieg zu einem Wirtschaftsfaktor in Kufstein wurde, wird durch diese Zahlen zusätzlich gestützt - natürlich unter der Annahme, dass dieses verfügbare statistische Material korrekt ist.

\section{Schluss}

In den vorangegangenen Kapiteln wurde deutlich, dass der Fremdenverkehr in Kufstein bereits vor dem Ersten Weltkrieg als Wirtschaftszweig sehr angesehen war und von den verschiedensten Vereinen entsprechend unterstützt wurde. Der Fremdenverkehr hatte sich im Laufe von rund vierzig Jahren in Kufstein wirtschaftlich etablieren können. Zu Beginn, ab ca. 1870, war es vor allem die Sommersaison, die für einen steigenden Zustrom von Gästen in der Stadt sorgte. Im Zeitraum 1904 bis 1914 entwickelte sich jedoch auch eine Wintersaison, die ebenfalls ein ökonomisch relevantes Wachstum verzeichnen konnte.

Die dieser Arbeit zugrundeliegende Forschungsfrage bezog sich auf die zentralen Entwicklungsschritte im Kufsteiner Fremdenverkehr und deren Beitrag zur Ausbil-

114 Die Quelle ist hier die jeweilige Fremden- und Wohnungsliste für Kufstein und das Kaisertal, welche in regelmäßigen Abständen während der Sommersaison vom Verein zur Hebung des Fremdenverkehrs herausgegeben wurde. Es handelt sich jeweils um die dort angegebene Gesamtzahl der Fremden für die Sommersaison.

115 Verein zur Hebung des Fremdenverkehrs, Kufstein, S. 58.

116 Die Gesamtgästezahl für die Jahre 1909 und 1910 sind der Dissertation von Runggaldier entnommen. Sie sind dort im Anhang für den Ort Kufstein angegeben, siehe: Runggaldier, Fremdenverkehr, Anhang S. 2.

117 Die Quellenangaben für diese Abbildung befinden sich in den obenstehenden Fußnoten, welche in der Abbildung integriert zu sehen sind. 
dung einer Sommer- und Wintersaison. Selbstverständlich stellt diese Arbeit keinen Anspruch auf Vollständigkeit hinsichtlich der Entwicklungsschritte, aber dennoch wurden wichtige Bereiche herausgearbeitet, die den Kufsteiner Fremdenverkehr gefördert und geprägt haben. Dabei stehen diese Entwicklungen oftmals eng miteinander in Verbindung. Kufstein lag verkehrsgünstig und konnte als Grenzstadt auf ein bereits bestehendes gewerbliches Gaststättenwesen zurückgreifen, als es zur gesellschaftlichen Trendumkehr in der Betrachtung der Bergwelt kam und diese als interessant und „bereisenswert" wahrgenommen wurde. Ohne den technischen Fortschritt durch den Bau der Eisenbahn wäre ein Zuwachs an Gästen vermutlich nicht in dem Ausmaß möglich gewesen. Für Kufstein spielte die leichte und raschere Erreichbarkeit mit der Eisenbahn nicht nur für die Sommersaison eine wichtige Rolle. Auch an den Zuwachsraten im Winter war die Eisenbahn beteiligt, als Sonderzüge die Teilnehmer*innen der Wintersportfeste nach Kufstein brachten. In der Person von Matthäus Hörfarter hatte Kufstein zudem einen touristischen Pionier, welcher die Zeichen der Zeit erkannte und den Fremdenverkehr geschickt zu unterstützen wusste. Die frühe Institutionalisierung der touristischen Förderung und die Positionierung Kufsteins als Kurort waren sein Vermächtnis für den Kufsteiner Fremdenverkehr. Er ebnete dadurch mit den Weg, dass Kufstein sich als Sommerziel etablieren und vom Fremdenverkehr wirtschaftlich profitieren konnte. Das Kaisergebirge und der Alpenverein sorgten für zusätzliche Attraktivität im Sommer und für steigende Gästezahlen.

Der Ausbau des Fremdenverkehrs im Sommer war der Grundpfeiler für die Begründung der Wintersaison: Das funktionierende und gut organisierte Beherbergungswesen sowie die Arbeit des Verschönerungs-, Fremdenverkehrs- und Alpenvereins trugen mit dazu bei, dass sich in der Stadt ein neuer Erwerbszweig mit ökonomischer Bedeutung entwickeln konnte. Kufstein, das diesen touristischen Wirtschaftsfaktor langfristig nutzen wollte, bemühte sich um den Ausbau der Saison. Als mit der Zunahme des Wintersports auch die Nachfrage nach einem Winterurlaub stieg, wurden sukzessive Investitionen in die touristischen Infrastruktur getätigt und so eine zweite Saison im Winter etabliert. Dass diese beginnende Wintersaison nicht einfach ein Zufall war, sondern bewusste und strategische Entscheidungen der Tourismusverantwortlichen vorausgegangen waren, zeigte sich unter anderem in der Gründung des Wintersportvereins oder in der Einführung der jährlichen Wintersportfeste. Und diese Strategie ging auf: Kufstein war einer der wenigen Orte in Tirol, denen es gelang, die Wintersaison vor dem Ersten Weltkrieg zu einem bedeutenden ökonomischen Faktor auszubauen. Vor allem aufgrund der sportlichen Wettkämpfe wurde der Winter zu einem wichtigen Werbeträger für den Ort und trug mit dazu bei, dass noch mehr Gäste auf Kufstein aufmerksam wurden. Die anhaltende Wintersportbegeisterung und Kufsteins Angebot an Sportstätten waren Erfolgsfaktoren für die beginnende Wintersaison vor 1914.

Die Anstrengungen der Stadt, im Winter eine zweite Saison aufzubauen, waren von Erfolg gekrönt. Denn gerade die Wintersaison sorgte dafür, dass die Kufsteiner Gesamtgästezahl vor 1914 beständig wachsen konnte. Die Zahlen, welche diesen Prozess bis 1910 belegen, wurden im vorhergehenden Abschnitt präsentiert und diskutiert. Ab 
dem Jahr 1911 wurden die Gästezahlen des ganzen Jahres vom städtischen Meldeamt erfasst. Waren es 1910 laut Runggaldiers Aufzeichnungen noch 26.624 Gäste im Gesamtjahr in Kufstein, so stieg die Zahl in den folgenden Jahren bis 1913 weiter an. Laut den Informationen im Artikel "Kufsteiner Fremdenverkehr in 60 Jahren", worin sich der Autor explizit auf die Angaben des städtischen Meldeamtes bezog, waren es 191128.000 Gäste, 191231.000 Gäste und 191331.485 Gäste jeweils im Gesamtjahr. Im Kriegsausbruchsjahr 1914 wurden in der Folge nur noch 24.514 Gäste in Kufstein gezählt. ${ }^{118}$ Ohne die beginnende Wintersaison wäre eine solche Steigerung der Gästezahlen nicht möglich gewesen, denn dann hätte sich die Anzahl an Sommergästen in Kufstein innerhalb von nur wenigen Jahren mehr als verdoppeln müssen.

Die Gesamtgästezahl in Kufstein gewinnt noch mehr an Bedeutung, wenn diese mit den Zahlen anderer Orte verglichen wird, beispielsweise mit den Südtiroler Orten, die auf eine längere touristische Vergangenheit und auf eine internationale Bekanntheit als Kurort zurückblicken konnten. Runggaldiers Zahlen der Orte mit mehr als 10.000 Fremden aus 1912 zeigen, dass Kufstein eine Gästefrequenz von Dreiviertel der Gästeanzahl von Meran (ohne Obermais) hatte und an vierter Stelle insgesamt nach Innsbruck, Bozen und Meran (ohne Obermais) bei den Tiroler Fremdenorten mit mehr als 10.000 Fremden lag. ${ }^{119}$ Diese beachtliche Gästefrequenz in Kufstein macht nochmals die wirtschaftliche Bedeutung des Fremdenverkehrs vor dem Ersten Weltkrieg deutlich.

\section{Literatur}

Beda Weber, in: Wikipedia. Die freie Enzyklopädie, aktualisiert am 21.12.2017, [https:// de.wikipedia.org/wiki/Beda_Weber], eingesehen 27.5.2018.

Bendler, Gebhard, Wilder Kaiser. Von Sommerfrischlern, Kletterlegenden, Skipionieren und dem Bergdoktor. 200 Jahre Alpingeschichte und Reisekultur, Innsbruck-Wien 2016.

Biasi, Franz, Kufstein. 600 Jahre Stadt 1393-1993, Innsbruck-Wien 1992.

Ders. (Hrsg.), Kufsteiner Buch. Beiträge zur Heimatkunde von Kufstein, Bd. 1 (SchlernSchriften 156), Innsbruck 1957.

Ders. (Hrsg.), Kufsteiner Buch. Beiträge zur Heimatkunde von Kufstein, Bd. 2 (SchlernSchriften 157), Innsbruck 1958.

Blattl, Josef, Kufsteiner Fremdenverkehr in 60 Jahren, in: Eduard Lippott (Hrsg.), 60 Jahre Tiroler Grenzbote 1871-1931, Kufstein 1931, S. 18-19.

Deutsche und Österreichische Alpenvereinssektion Kufstein (Hrsg.), Die Erschließung des Kaisergebirges. Nach Aufzeichnungen in Hinterbärenbads Tourenbüchern bearbeitet von F. Nieberl, Kufstein $1912^{2}$.

118 Blattl, Fremdenverkehr, S. 18.

119 Runggaldier gibt folgende Zahlen für 1912 in seiner Tabelle „Fremdenorte mit mehr als 10.000 Fremden" an: Innsbruck 192.681, Bozen 108.980, Meran 38.943, Kufstein 30.520, Landeck 15.430, Brixen 11.885, Runggaldier, Fremdenverkehr, Anhang S. 2. Worin die Abweichung bei Kufstein zu den Zahlen von Blattl besteht, ist leider nicht ersichtlich. Allerdings besteht die Vermutung, dass Blattl in seinem Artikel eventuell großzügig auf die gerade Zahl 31.000 aufgerundet hat. 
Forcher, Michael, Zu Gast im Herzen der Alpen. Eine Bildgeschichte des Tourismus in Tirol, Innsbruck 1989.

Hachtmann, Rüdiger, Tourismus-Geschichte, Göttingen 2007.

Heimatverein Kufstein, Rodeln vor 100 Jahren, o. D., [http://www.heimat-kufstein. at/2012/35-rodeln-vor-100-jahren und http://www.heimat-kufstein.at/bildergalerie/ category/38-0113-rodeln-v-100-jahren], eingesehen 15.10.2018.

Heinrich Noë, in: Wikipedia. Die freie Enzyklopädie, aktualisiert am 2.10.2018, [https:// de.wikipedia.org/wiki/Heinrich_Noë], eingesehen 10.10.2018.

Hotel Gisela Kufstein, o. D., [https://www.hotelgisela.at/], eingesehen 16.08.2018.

Kuntscher, Herbert, Wintersport seinerzeit, in: Rotary Club Kufstein/Verein für Heimatkunde und Heimatschutz Kufstein (Hrsg.), Kufstein. Bayerns Glanzperle Tirols, Kufstein 2012, S. 168-175.

Kyselak, Joseph, Zu Fuss durch Österreich. Skizzen einer Wanderung nebst einer romantisch pittoresken Darstellung mehrerer Gebirgsgegenden und Eisglätscher unternommen im Jahre 1825 von Joseph Kyselak, nachgegangen und nachgedacht von Ernst Gehmacher, Wien u. a. 1982.

Lässer, Adolf, 100 Jahre Fremdenverkehr in Tirol. Die Geschichte einer Organisation (Tiroler Wirtschaftsstudien 40), Innsbruck 1989.

Leonardi, Andrea/Heiss, Hans (Hrsg.), Tourismus und Entwicklung im Alpenraum 18.20. Jh. (Tourism \& Museum, Studienreihe des Touriseum 1), Innsbruck u. a. 2003.

Lippott, Eduard (Hrsg.), 60 Jahre Tiroler Grenzbote 1871-1931, Kufstein 1931.

Ders., Kufsteiner Chronik 788-1918, in: Franz Biasi (Hrsg.), Kufsteiner Buch. Beiträge zur Heimatkunde von Kufstein und Umgebung, Bd. 2 (Schlern Schriften 157), Innsbruck 1958, S. 9-142

Lunglmayr, Alfred, Drei Sommer in Tirol, in: Der Alpenfreund. Illustrierte Touristenzeitschrift für das Alpengebiet 2 (1896), Heft 12, S. 1-4.

Pelinka, Anton/Maislinger, Andreas (Hrsg.), Handbuch zur neueren Geschichte Tirols (Zeitgeschichte 2), Innsbruck 1993.

Planer, Klaus, Der Fremdenverkehr Nordtirols, Diss. Wien 1970.

Riedmann, Josef, Geschichte Tirols, Wien $1988^{2}$.

Rotary Club Kufstein/Verein für Heimatkunde und Heimatschutz Kufstein (Hrsg.), Kufstein. Bayerns Glanzperle Tirols, Kufstein 2012.

Runggaldier, Engelbert, Tirol und der Fremdenverkehr, Diss. Innsbruck 1927.

Sandgruber, Roman, Die Entstehung der Österreichischen Tourismusregionen, in: Andrea Leonardi/Hans Heiss (Hrsg.), Tourismus und Entwicklung im Alpenraum 18.-20. Jh. (Tourism \& Museum, Studienreihe des Touriseum 1), Innsbruck u. a. 2003, S. 201-226. 
Ders., Ökonomie und Politik. Österreichische Wirtschaftsgeschichte vom Mittelalter bis zur Gegenwart (Österreichische Geschichte), Wien 1995.

Sinwel, Rudolf (Hrsg.), Dr. theol. Matth. Hoerfarter. Gedenkschrift zur Enthüllung des Hoerfarter-Denkmales in Kufstein am 10. September 1899, Kufstein 1899.

Tourismusverband Kufsteinerland, Geschäftsbericht 2017, 2017, [https://www.kufstein. com/media/geschaeftsbericht-tourismusverband-kufsteinerland-2017.pdf], eingesehen 5.6.2018.

Tschurtschenthaler, Paul, Der Tourismus im Bundesland Tirol 1918-1990, in: Anton Pelinka/Andreas Maislinger (Hrsg.), Handbuch zur neueren Geschichte Tirols (Zeitgeschichte 2), Innsbruck 1993, S. 113-208.

Türkis, Benjamin, Innsbrucker Tourismusgeschichte (Geschichte und Ökonomie 20), Innsbruck 2010.

Weitlaner, Paul, Dekan Dr. Matthäus Hörfarter, in: Franz Biasi (Hrsg.), Kufsteiner Buch. Beiträge zur Heimatkunde von Kufstein und Umgebung, Bd. 1 (Schlern Schriften 156), Innsbruck 1957, S. 121-138.

\section{Quellen}

Baedeker, Karl, Südbayern, Tirol, Salzburg, Ober- und Nieder-Österreich, Steiermark, Kärnten und Krain. Handbuch für Reisende, Leipzig $1912^{35}$.

Fremdenverkehrs-Verein Kufstein (Hrsg.), Kufsteiner Fremden-Liste, Kufstein 1905-1910.

Postkarte, 5.10.1937. Heimatverein der Stadt Kufstein, Fotosammlung Kufstein.

Prospekt Gasthof zum „Auracher“, o. D. (nach 1895). Innsbruck.

Prospekt Hotel Egger (Familie Greiner), O. D. (nach 1909). Hannover-Ricklingen.

Sektion Kufstein des Deutschen und Österreichischen Alpenvereins (Hrsg.), Hinterbärenbad im Kaiser-Gebirge. Ein Geleit- und Erinnerungs-Büchlein für KaisergebirgsFreunde, Kufstein 1904.

Verein zur Hebung des Fremdenverkehrs in Kufstein und Umgebung (Hrsg.), Kufstein und seine Umgebung. Ein Führer für Fremde und Einheimische, Kufstein 1904.

Verein zur Hebung des Fremdenverkehrs in Kufstein (Hrsg.), Fremden- \&Wohnungsliste für Kufstein und das Kaisertal, Kufstein 1895-1904.

Wintersportvereinigung Kufstein (Hrsg.), Winter in Kufstein, München 1911. 
Tanja Angela Braunschmid ist Absolventin des Bachelorstudiums Geschichte an der Universität Innsbruck. tanjabraunschmid@hotmail.com

\section{Zitation dieses Beitrages}

Tanja Angela Braunschmid, Die Entwicklung des Fremdenverkehrs in der Stadt Kufstein bis 1914. Sommerfrische, Bergsport, Kur und die Anfänge des Wintertourismus, in: historia.scribere 12 (2020), S. 159-188, [http://historia.scribere.at], eingesehen 15.6.2020 (=aktuelles Datum).

(C) Creative Commons Licences 3.0 Österreich unter Wahrung der Urheberrechte der Autorlnnen. 